\title{
Wait and see? \\ Public opinion dynamics after terrorist attacks
}

May 21, 2021

\begin{abstract}
We use the occurrence of the London bombings in July 72005 during the fieldwork period of the British Social Attitudes Survey to analyse the dynamics of public support for measures curbing core freedoms. We observe little changes in public attitudes towards counter-terrorist policies in the first week after the attack. Approval of infringements on privacy and procedural rights surges in the following weeks before stabilizing at an increased level in the medium run. Our findings indicate that, when it comes to restrictive counter-terrorist measures, the public adopts a wait-and-see attitude rather than reacting in fear. Ancillary analyses suggest that the media may be the source of attitudinal changes on policies curtailing civil liberties.
\end{abstract}

Word count: Approx. 9,850 words 
"Arbitrary arrest, indefinite detention without trial, "rendition", suspension of habeas corpus, even torture - who would have thought such things possible?", asked The Economist in 2007. In virtually all liberal democracies, a vast array of liberticidal measures has been used as means to respond to the rise of international terrorism (Epifanio, 2011; Neumayer et al., 2014). Like total war, terrorism calls for revising the complex balance between the need of enhancing security and that of limiting restriction of civil rights in favor of the former priority. Unlike wars, however, terrorism is potentially endless. For this reason, while the suspension of these fundamental rights is viewed as necessary in times of threat, many worry that Western democracies might be enduring a permanent restriction of civil liberties.

How did we get there? Many scholars have documented the demand by the public for greater security even at the cost of widespread infringements on individual rights. For example, in the USA, the counterterrorist measures implemented during the Presidency of George W. Bush as an emergency response to the September 11, 2001 terrorist attacks were popular at the time and are still supported by a large share of the American public today (Mueller and Stewart, 2018; Brooks and Manza, 2013).

When and how do people change opinion on civil liberties after a terrorist attack? How much of this reversal of attitudes on individual rights is due to public fear? What is the role of elites, such as politicians and the media, in shaping the demands for security measures? To make some progress in tackling these questions, this paper studies the dynamics of public reactions to a terrorist shock. We exploit the occurrence of the London bombings in July 7, 2005 (7/7) during the fieldwork period of the British Social Attitudes Survey. The terrorist attack occurred right at the time when most respondents were being interviewed. This allows us to follow week by week how a terrorist event changes public attitudes on illiberal policies.

Our results challenge the notion of an over-responsive public driven by emotions when it comes to core individual rights. In the first week after the attacks there is a small increase in support for policies curtailing fundamental freedoms, though this effect is not statistically significant. Public opinion does change, and significantly so, but with a delay. The public becomes more likely to approve security enhancing policies that reduce privacy rights and procedural guarantees for terrorist suspects in the following weeks. These demands for more security at the expense of civil freedoms then stabilize at increased levels in the medium run. These results are suggestive of a public adopting a more "reasoned" approach to trade-off between liberty and security. We 
therefore investigate the role of the elite in shaping public attitudes. We find little evidence in favor of politicians' influence. Changes in public stances match the discourse of the media.

The British Social Attitudes Survey in the 2005 edition was carried out between May and November, with the bombings hitting London during the main data collection period. Unlike previous and next editions, the survey included questions on support for counter-terrorist policies that are intended to minimize the risk of future attacks at the cost of harming individual freedoms. If implemented, these measures cut back privacy rights (e.g. tapping phone and email conversations), curb freedom of expression (e.g. banning free speech or demonstrations) and curtail the legal rights of terrorist suspects (e.g. arbitrary detention, fair trial and torture).

We compare respondents' demands for counterterrorist measures before and after the attack. One key contribution is that we monitor changes in attitudes in the very short run (first week after the attack), short run (first month minus the first week), and medium run (second month till the end of the survey). In tracking public opinion dynamics over three time periods, we seek to monitor, as much as the data permit, the emotional and the reasoned responses to the terrorist attack. Under the assumption that the date of the interview for each respondent is random (balance tests provide evidence in support for this assumption), our empirical strategy comes close to uncovering the causal effect of a terrorist shock.

As noted above, the dynamics of public stands on civil liberties exhibit a weak reaction to the terrorist attack in the first week after the bombings. The public becomes more prone to cut back individual freedoms in the weeks that followed. This effect is substantial, equal to approximately $40 \%$ of a standard deviation, enough to move the average respondent form relatively opposed to relatively in favor of restrictive policies. The picture looks very different when we consider the effect of the attack on other items which are often associated with emotions. In the aftermath of the bombings, respondents are more permissive of aggressive military actions, and report higher levels of trust towards other people, all in the very short run. All these effects fade away in short and medium run. While public support for security measures increases with a delay, terrorist threat perception increases immediately after the terrorist attack, and stay high throughout the whole survey period. The dynamic of support for security measures is, thus, unique among the survey questions we study. This prompts us to investigate why we observe such a delayed shift in public attitude.

We look at two possible sources of influence: politicians and the media. While we cannot fully rule out that the former are at the origin of the opinion shift on liberticidal policies, we find 
little support for this hypothesis. Respondents are unaffected by a well-publicized speech on antiterror measures delivered in August 5, 2005 by then Prime Minister, Tony Blair. In addition, we document that only respondents who voted Conservative in the last legislative elections in May 2005 are willing to curb core freedom following the London bombings. This suggests that the government was unable to persuade its main constituency, Labour voters.

To test for a possible effect of the media, we conduct a text analysis of press coverage on terrorism, civil liberties and counter-terrorist policies before and after the bombings. Our findings show that the first week after the attack is dominated by a description of the events that unfolded on $7 / 7$. A discussion of counter-terrorist policies, in turn, became more pronounced in the short and medium run, in particular when it comes to the popular press (the Sun, the Daily Mirror, and other tabloids). While these results are only descriptive, the variation in media coverage matches relatively well the dynamics of public opinion we uncover. As such, media persuasion appears as one of the sources of the changes of attitudes in the short run after the attacks.

This paper is structured as follows: Section 1 frames public responses to terrorism within the arguments and findings of the scholarly contributions in the field. We describe the 2005 London attacks in Section 2. We present our data and identification strategy in Section 3 and we describe our main results in Sections 4. Section 5 discusses the possible mechanisms behind our findings, and the last section concludes.

\section{Policy demands following terrorist attacks}

A large literature uses the occurrence of terrorist attacks during the field work period of attitudes surveys to uncover the causal impact of terrorism on public opinion 11 This research design has been adopted to study the effect of terrorism on items as diverse as voting intentions (Balcells and Torats-Espinosa, 2018), political attitudes (Castanho Silva, 2018), attitudes towards minorities and discrimination (Nägel and Lutter, 2020; Boydstun et al., 2018; Echebarria-Echabe and Fernandez-Guede, 2006; Giani, 2020; Jakobsson and Blom, 2014; Giani and Merlino 2020), restric-

\footnotetext{
${ }^{1}$ Many other methodological approaches have been employed. Some contributions have been looking at the effect of terrorism on economic and behavioral outcomes (Gautier et al., 2009; Prager et al. 2011); others have studied voting outcomes (Getmansky and Zeitzoff, 2014; Montalvo, 2011). A number of works have been concerned with attitudes towards migrants or of migrants themselves (Böhmelt et al., 2020; Van de Vyver et al., 2016; Hanes and Machin, 2014; Elsayed and De Grip, 2013). Of most relevance for us is Economou and Kollias (2018) who compare attitude on security policies in EU countries around half a year after the terrorist attacks in France in 2015. Finding no difference in support for counter-terror policies in France and other countries, they conclude that the effect in favor of security measures is short lived.
} 
tive immigration policies (Boomgaarden and de Vreese, 2007; Finseraas et al., 2011, 2013; Nussio et al., 2019; Solheim, 2019; Legewie, 2013), refugees (Breton and Eady, 2021) or trust broadly conceived (Nussio, 2020; Van Hauwaert and Huber, 2020; Geys and Qari, 2017; Arvanitidis et al., 2016; Dinesen and Jæger, 2013; Metcalfe et al., 2011; Perrin and Smolek, 2009). In contrast to these studies, our analysis focuses on public opinion stances on core individual freedoms. We are not unique in that respect. Like us, Bozzoli and Müller (2011) also rely on the 2005 British Social Attitudes Survey to study the relationship between perception of threats and demand for greater security using the London bombings as an instrument for respondents' assessment of the risk of terrorist attacks. Unlike us, they are not interested in the dynamics of public opinion and what they mean for our understanding of the increased demand for security policies after terrorist attacks.2 $2^{2}$ We believe, however, that these dynamics are critical to better understand how strong restrictions on civil liberties came to be accepted and supported by a large part of the population. To explain why, we first outline the divide in the literature on the psychology of citizens following terrorist events. As an exhaustive overview of these important scholarly contributions is beyond the scope of this paper, we only briefly describe the debates most relevant for our purpose.

Terrorism is often believed to generate an emotional response. This reaction is usually linked to the "psychological effects of terrorism on audiences" (Crenshaw, 1986: 400), with threat as the main catalyst of individuals' reactions. The so called "bath tub fallacy" is often evoked to describe the volatility of public opinion about low-probability events (Muller and Stewart, 2018; Viscusi and Zeckhauser, 2003) for which panic, shock, and fear dominate over reasoned appraisals. Even though the risk of dying out of terrorism is certainly lower than from other types of hazards, such as slipping and dying in a bathtub, terrorism triggers emotional responses that generate a "probability neglect" (Sunstein, 2003). These emotional reactions also open a window of opportunity for policymakers to push forward their security agenda. As Sunstein (2003: 122) puts it, after a terrorist attack, the public "alters its behavior and demand substantial government response (...) that does not reduce the risk and might in fact make things worse" (Sunstein, 2003: 122).

A host of empirical papers seek to distinguish the emotions generated by a terrorist attack and their likely effects on public attitudes towards counterterrorist measures. Feeling of personal threats, unsurprisingly, have been shown to play a key role. Huddy et al. (2005) conduct a survey of American citizens in the months following $9 / 11$ and find that perceived threat is the key driver

\footnotetext{
${ }^{2}$ Bozzoli and Müller briefly depict the timing of respondents' average answers to some question items and call for a better understanding of these dynamics, which this paper tries to provide.
} 
behind the demand for foreign interventions and the support for Bush's anti-terrorist agenda. Relatedly, Merolla and Zechmeister (2009) experimentally manipulate subjects' perception of threat, via news story, either in video or print format. The authors find that, under conditions of anxiety and fear, individuals become less tolerant of perceived outgroups (immigrants and gay people), call for strong political leaders, and are willing to trade civil liberties and become more supportive of aggressive foreign policies. Anger as primary motivator of offensive policies is also found in several other studies, with angry individuals more likely to favor punitive measures compared to fearful respondents, who are instead keener to endorse conciliatory policies (Giner-Sorolla and Maitner, 2013; Lerner et al., 2003). But emotions do not have to be "egotropic" or negative. Sociotropic concerns, namely a perception of threat to the whole society, rather than personal concerns, are often regarded as predictors of public support for anti-terrorist measures (Davis, 2007; Davis and Silver, 2004; Joslyn and Haider-Markel, 2007, 2018). Pride and hope have been associated with an increase support for counter-terrorist efforts and higher levels of government's trust or support for incumbent politicians, the so called rally-around-the flag effect (Gross et al., 2009; Davis and Silver, 2004; Hetheringhton and Nelson, 2003; Kam and Ramos, 2008; Merolla and Zechmeister, 2009; Perrin and Smolek, 2009; Willer and Adams, 2008).

Overall, this literature emphasizes the role played by feelings and how they shape people's demands for counterterrorist measures (even when scholars document a negative relationship such as anxious people being less likely to endorse military actions abroad and to back President Bush's counterterrorist actions, see Huddy et al., 2005). As Muller and Stewart argue (2018: 1), public opinion is "the primary driver behind the extensive and excessive counterterrorism efforts undertaken since $9 / 11$, and officials and elites are more nearly responding to public fear than creating it".

If public reactions to terrorist attacks are driven mostly by emotions, shifts of attitudes towards restrictive policies should be stronger in the short run compared to the long run. Such a pattern is documented by Davis (2007) who shows lower support for liberticidal measures in 2003 and 2004 than in 2001. In our context, we would expect (on a shorter time frame) a similar pattern. That is, if emotions are the main drivers of public responses, changes in public attitudes on security measures should be weakly stronger in the very short run (first week after the attack) than in the short (first month) and medium run (first quarter).

Not all scholars agree on the assumption of a panic prone public, who emotionally overreacts to a terrorist attack. For some, citizens exhibit, what we call, a "reasoned response" to terrorism. 
The medical literature frames community responses to terrorist events within a three phases model. In the immediate aftermath of an attack, individuals are more likely to be stunned, numbed or in denial, with only a minority experiencing panic. In the "recoil phase", people tend to process information and make sense of what has happened. Finally, in the recovery phase, individuals adjust to the new state by showing a high level of resilience (Tyhurst, 1951 in Alexander and Klein, 2005). Sheppard et al. (2006) draw on case studies, including the USA terrorist attacks in 2001 and the 2005 London bombings, to question the belief that people react irrationally in times of emergency. The authors contend that in the immediate aftermath of a terrorist shock the public is "fairly resilient, calm and rational in its reactions" (2006: 238). This finding is in line with Bux and Coyne's (2009) conclusion that a significant part of the public is resilient and able to cope emotionally in the aftermath of a terrorist attack. In dispelling the conventional wisdom of a panic prone public, this literature points to the possibility that the public reacts to governmental and media messages. Perry and Lindel (2003) as well as Rogers et al. (2007) optimistically argue in favor of government communication about the threat as a way to minimize the social and psychological impact of terrorist attacks. Brooks and Manza (2013), more depressingly, show that the wording of policy options can affect respondents' support for various liberticidal measures. In all, the public

reacts not to the attack, but to the information provided to them. Building on this literature, if the public is unlikely to emotionally over-react to terrorism, we should expect no shifts in public attitudes in the very short term (first week). Changes in attitudes, if any, should be observed with delay once individuals assess the risk and gather information on the nature and extent of the terrorist exposure.

The dynamics predicted by "emotional" and "reasoned" responses are quite distinct. Of course, these two opposite poles can be considered as "ideal types". In practice, both can coexist within an individual. What this paper investigates is which type of response dominates, on average. To do so, we use the 2005 British Social Attitudes Survey and the London bombings in July 7, which we now briefly describe.

\section{July 2005: the London bombings}

On July 6, 2005, scenes of jubilation could be seen in different parts of London, with people celebrating the International Olympic Committee's decision to award the 2012 Olympic Games to the UK capital. The day after, Thursday 7 July, images of terror colonized the scene. People travel- 
ing that morning on the London transportation system were the target of one of the most deadly terrorist attacks experienced by the UK. At 8.50 am within one minute, three bombs exploded on the Circle line near Aldgate and at Edgware Road stations, and on the Piccadilly line near Russell Square. Less than one hour later, a double-decker bus burst in Tavistock Square. Fifty two London residents died in the blasts and seven hundred reported serious injuries. The bombings, carried out with homemade explosives, were perpetrated by four men, all radical Islamist terrorists, and all British citizens, who blew themselves up in the attacks.

It was clear, already by midday, that London just experienced an act of terrorism, and of Islamist nature. The identity of the perpetrators became known only few days later but just within few hours from the blasts the "Secret Organisation Group of Al Qaida in Europe" claimed responsibility for the attacks on the internet $]^{3}$ "I have no doubt whatsoever that this is a terrorist attack" confirmed the Mayor of London at that time, Ken Livingstone. $4^{4}$ Former Foreign Secretary, Jack Straw, corroborated this statement by adding that the bombings had the "hallmarks of an al-Qaeda-related attack." "5 Coming back from Scotland, where the summit of the G8 countries was taking place, the then UK Prime Minister, Tony Blair acknowledged that "this is a terrorist attack or series of terrorist attacks; it's also reasonably clear that it is designed and aimed to coincide with the opening of the G8." ${ }^{6}$ By the late afternoon, the whole international community joined the UK in condemning the barbaric attacks "without reservation", with the UN Security Council unanimously adopting resolution 1611 aimed at combating terrorism with "utmost determination." 7

Dramatic images of the underground and the bus after the blasts, footage of critically injured people, eyewitnesses' accounts of the horrific bombings, live pictures of traffic CCTV cameras, and reconstructions of the timeline of the attacks were transmitted by virtually all media channels throughout 7 July. The BBC Online website recorded a peak of 40,000-page requests per second and some 1 billion total accesses. The day after, images of the remains of the exploded doubledecker bus, decimated tube trains, bomb victims covered in blood and passengers led away from the blast scene were in the front pages of virtually all newspapers in the UK and abroad. The Guardian's headline read "London's day of terror," the Daily Telegraph chose "Al-Qa'eda brings terror to the heart of London", and the Daily Star preferred the laconic "Bastards." 8

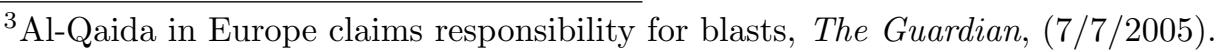

4 Text of statement by Mayor Ken Livingstone, Financial Times, (7/7/2005).

$5 \overline{B B C,(7 / 7 / 2005) \text {. }}$

${ }^{6}$ Transcript of Tony Blair speech, $C B C$ News Online, $(7 / 7 / 2005$. $)$

7 Security Council, Press Release, (7/7/2005).

${ }^{8}$ What the papers say, The Guardian, (8/7/2005).
} 
Two weeks after the horrific attacks on the 7th of July, London was under siege again. On Thursday 21 July, commuters on the Hammersmith \& City, Northern and Victoria undergrounds lines and the number 26 bus witnessed a number of explosions. These blasts were small and no injury or death were reported. It became known in the following day that only the detonator caps of the devises exploded while the bombs themselves did not blast. It also became known that four suspects, all Islamist extremists, were wanted in connection with the attacks and eventually arrested on July 29th.

The first explicit and public announcement of the measures the government was determined to implement as a response to the terrorist attacks came on the 5th of August. The then PM Tony Blair called a press conference to warn that "the rule of the game are changing" 9 He outlined twelve counterterrorist measures to be taken "immediately, or under urgent examination", ranging from extradition, deportation and revocation of citizenship for terrorist suspects to the extension of pre-charge detention, increased powers of security authorities and new offenses for glorification of terrorism 10

This brief reconstruction of the events of the 7th of July and the events unfolding in the days and weeks that followed highlights three important features of the London bombings. First, the attacks came as a surprise; second there was very little uncertainty about the nature of the attacks, especially their link with Islamist terror. Third, the bombings increased the salience of terrorism in the eyes of the British public. Thus, $7 / 7$ is a good setting to understand how public opinion responds to terrorism. We now turn to this question using the British Social Attitudes Survey.

\section{Empirical analysis}

\subsection{The Survey}

The British Social Attitudes Survey $(B S A S)$ is intended to produce annual measures of attitudinal changes on a number of social issues, ranging from public spending and social welfare to disability, social identity and prejudice, inter alia. The survey is designed to yield a representative sample of the population in the UK aged 18+. Household addresses are drawn from Postcode Address File, and one person in the household is interviewed. To account for the fact that people in small households have a higher probability of selection than people in large households, the data are

\footnotetext{
9" "The rules of the game are changing", The Guardian, (5/8/2005).

${ }^{10}$ Full text: The prime minister's statement on anti-terror measures, The Guardian, (5/8/2005).
} 


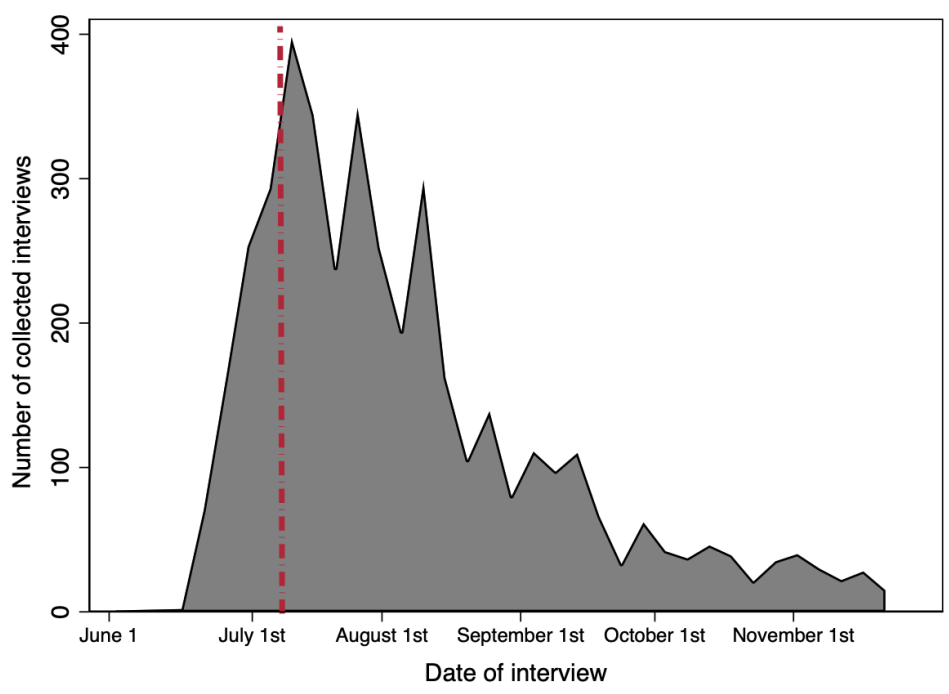

Figure 1: Data collection around the terror attack. The red spike corresponds to the date of the attack. On July 7, four Al-Quaeda suicide bomber detonated four bombs in public transports, killing 52 people.

weighted ${ }^{11}$ Interviews are conducted face-to-face and, in the 2005 edition, lasted about 65 minutes followed by a self-completion questionnaire.

We use this survey for two reasons. First, the 2005 round of the $B S A S$ was carried out between the 31st of May and the 24th of November, with most of the data collected between June and September. The London July 7th attacks, thus, occurred during the main fieldwork period and did not interrupt the running of the interviews. Figure 1 plots the daily distribution of completed surveys. Second, unlike previous and subsequent editions, the $2005 B S A S$ included a set of questions on public support for various restrictive counterterrorist measures. The relevant survey items contain specific reference to extrajudicial practices, such as arbitrary detention and torture, and limitations of privacy rights, such as wiretapping, censorship, banning of demonstrations, and free speech. The BSAS goes to a great lengths to avoid wording effects by incorporating a detailed pre and post formulation for each of the question on the topic (see Appendix $\mathrm{B}$ for the wording of these survey items).

\subsection{Identification strategy and potential issues}

For exposition purposes, as well as to avoid multiple hypothesis testing problems, we use principal component analysis to construct our main dependent variables, denoted $y_{i}$. In one regression,

\footnotetext{
${ }^{11}$ For a full description of the $2005 B S A S$ and the technical details of the Survey, see Park et al. 2007. British Social Attitudes: The 23rd Report.
} 
our dependent variable is Privacy rights, an index constructed by using the first component of four survey items, which refer to policy demand for banning protests and free speech, controlling emails and imposing a compulsory ID card. In a second regression, our dependent variable is Procedural rights, an index constructed by using the first component of four different survey items, including the policy demand for arbitrary detention, torture, the possibility of tagging suspect without mandates, and the denial of a jury in trials. The weights on each component of both indexes vary from 0.41 to 0.57 (based on pre-treatment observations) and are barely affected by the treatment (see Appendix $\mathrm{D}$ for more details). This means that our results are unlikely to be driven by one of the components of the indices.

The treatment variable $T_{i} \in\{0,1\}$ takes value 0 if the respondent was interviewed before the attack and 1 if the respondent was interviewed after the attack. Our empirical approach compares answers between control (pre-attack) and three different treated groups, interviewed at three distinct intervals of time. We first compute the treatment effect among respondents interviewed within 7 days after the attack (from $8 / 7$ to $14 / 7$ ), the very short run. In this time period we seek to assess public reaction when the threat is the most salient, emotions are potentially high, and policy responses are yet to be proposed. We then focus on respondents interviewed in the first month (minus the first week) of the attacks (from 15/7 to 6/8), the short run. We think of the short run as the interval during which, after the initial emotional reaction, the media and politicians start talking about counter-terrorist initiatives. Finally, we look at the responses of individuals interviewed in the second month till the end of survey (from $7 / 8$ to 24/11), the medium run. This allows us to understand whether any reaction is short-lived or yields a more permanent shift in attitude.

The analysis controls for a set of covariates, summarized by $X_{i}$; that include income (0-14), education (0-7), sex (0-1), age (18-99), age-squared, whether the respondent lives with children $(0-1)$ and whether she has immigration background (0-1). Finally, accounting for the fact that responses to terror attacks might be mitigated by the incumbent's partisanship (i.a. Merolla and Zechmeister, 2013), we add a dummy taking value 1 if the respondent voted Labour in the 2005 election in the UK. Controlling for region fixed effects, we estimate the treatment effect with OLS according to the following specification:

$$
y_{i}=\alpha+\beta T_{i}+\gamma^{\prime} X_{i}+\epsilon_{i}
$$


where $\beta$ is the main coefficient of interest and $\epsilon$ is the error term.

Muñoz, Falcó-Gimeno and Hernández (2020) suggest that the causal interpretation of research designs using unexpected events is warranted when the shock is unexpected and salient, it does not interrupt the running of the survey, there are no issues of imbalances due to quota sampling nor problems of reachability and non-compliance. In our setting, the 2005 London attacks came unexpectedly and were highly salient, as described above. There is no mention of any disruption in the running of the British Social Attitudes Survey in coincidence with or after the 7/7 attacks (Park et al., 2007). Similarly, the $B S A S$ is not based on quota sampling and the timing of interviews is as good as random. While we cannot completely exclude some differences between control and treatment group, we take a number of steps to mitigate these concerns. First, the very short run (July 8 to July 14) and the short run (July 16 to August 6) correspond to the periods when most of the interviews took place (see Figure 1). This is also the period when the terrorist attacks were most likely to be on the mind of the voters, allowing us to minimize the influence of external events. Second, we conduct a number of robustness tests, which reinforce our findings. We account for the possible unbalanced distribution of socio-economic covariates on control and treated units by detecting and pruning outliers through pretreatment matching. We then run the same analysis without outliers, using coarsened exact matching (Iacus et al., 2012). Results can be found in Appendix E, We also weight the control group in a way such that the moment conditions of each covariate match the one of the treatment group, using entropy weighting (Hainmueller, 2012). Appendix G shows the empirical estimates associated with this specification. Despite the potential issue of multiple hypothesis testing, for completeness, we also look at the impact of the London bombings on each item composing the privacy and procedural rights indexes separately. Our results are very consistent across all dimensions (see Appendix F). Since the survey is stratified and some respondents interviewed in the last weeks may have different characteristics, we perform our analysis using different end dates for the medium run (which consists of all observations from August 7 to the end of the survey in our baseline presented in the main text). Results remain the same however we define the medium run (results available upon request). Finally, we conduct a number of placebo tests, changing the time of the attacks and using survey items on issues (government spending in healthcare), which should not be affected by the terrorist bombings (Figure G.1). 


\section{Results}

In this section, we present our empirical findings building on our discussion above of the existing literature. We first report the results on the dynamics of public support for restrictive counterterrorist policies. We then compare our findings with the evolution of public stances on survey items usually linked to emotional reactions.

\subsection{Dynamics of public support for counter-terrorist policies}

Figure 2 displays the treatment effects for privacy and procedural rights in very short run, namely the first week after the London bombings (from July 8 to July 14, 2005), the short run (from 15/7 to $6 / 8)$ and the medium run $(7 / 8$ to $24 / 11)$. Regression coefficients are displayed in Table E.1 in Appendix E.

The left panel displays the effect for privacy rights. We observe a positive, but relatively small change of attitudes in the very short run. The increase in support for restrictions becomes more pronounced and statistically significant in the short run. The effect is substantial. The demand for privacy-reducing counter-terror policies increased by about $18.8 \%$ relative to the pretreatment mean, significant at $p<.01$ in the short run (point estimates are displayed in Table E.1) 12 The demand for security stabilizes in the medium run, remaining significant at $1 \%$.

The right panel shows the impact of the terrorist attacks on procedural rights. We notice a relatively weak effect of the London bombings in the very short run. The public becomes keener to accept curtailments of procedural rights in the short run, with a treatment effect of about $21.0 \%$ relative to the mean, statistically significant at $1 \%$. In the medium run, the treatment effect remains significant at $p<.01$. However, we observe a mild decrease compared to the short run: the increase in support is about $13.6 \%$ relative to the mean. Still, the difference between the estimates for the short and medium runs is not statistically significant.

The patterns in Figure 2 suggest a delay in public shifts towards restrictive policies. Support for restrictive measures slightly increases in the first week after the bombing, becomes more pronounced in the short run, stabilizing afterwards (albeit, with a slight decline for procedural rights). This dynamic runs counter to the idea of an emotional public. As we noted above, if emotions drive the response after the attacks, we should expect a sharp increase in the very short run, followed

\footnotetext{
${ }^{12}$ The value of the estimated treatment effect is $\hat{\beta}=.59$. Re-scaling our index for privacy right to have a minimum at zero, the control group takes the value of 3.13 . Hence, the treatment effect adds up $.59 / 3.13 \approx 18.8 \%$. Percentage effects reported below are computed using the same methodology.
} 
Figure 2: Treatment effects for Privacy and Procedural Rights
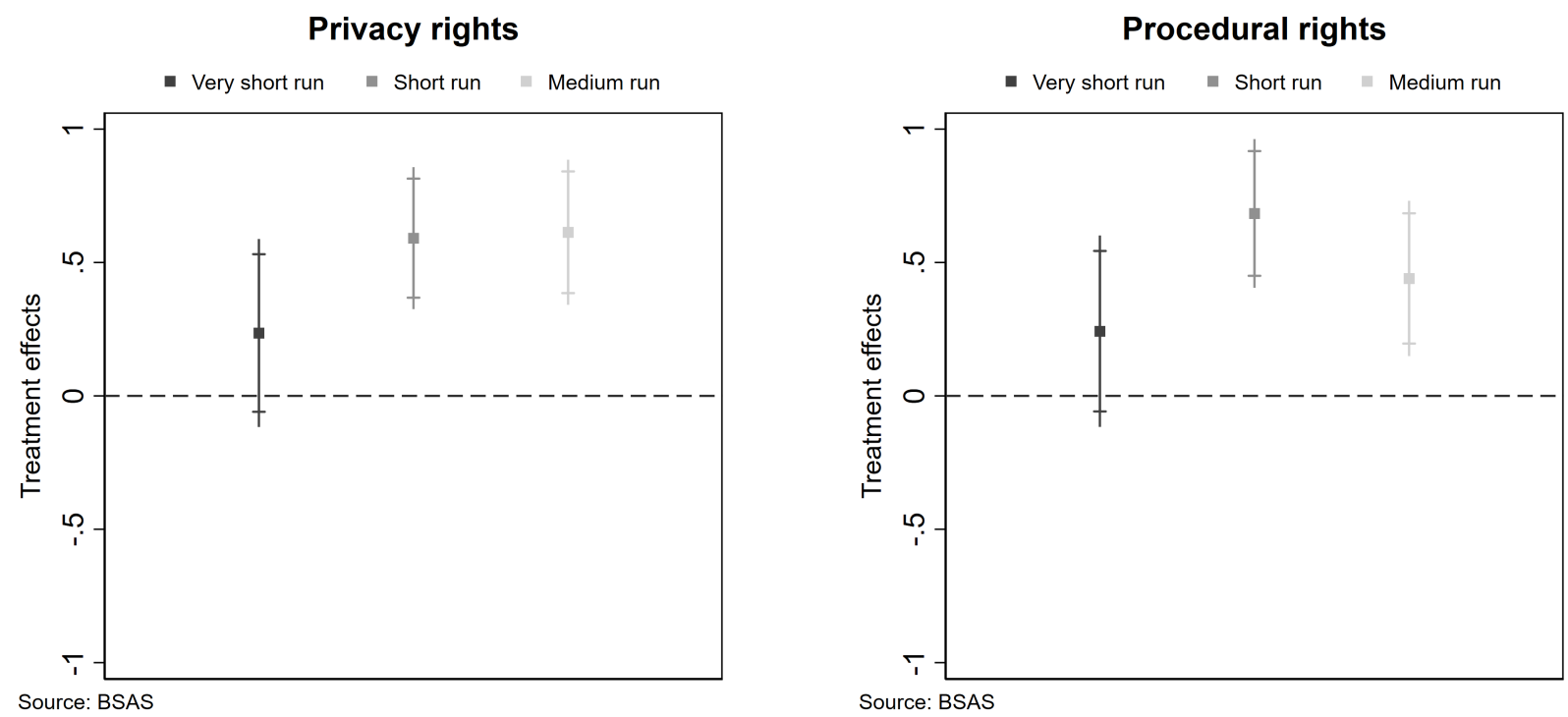

Notes. In both figures, spikes refer, respectively, to very short run, short run and medium run. Confidence intervals at $95 \%$. Privacy and procedural rights are constructed using the first component of a principal component analysis discussed in Section 3.2 Treatment effects are estimated using OLS, controlling for income (0-14), education (0-7), sex (0-1), age (18-99), age-squared, whether the respondent lives with children (0-1), whether she has immigration background (0-1), whether the respondent voted Labour during the 2001 election in the UK as well as region fixed effects.

by a decrease in the weeks after. Instead, the observed variations are suggestive of a wait-and-see approach, more consistent with a reasoned response and in line with the three phases identified in the medical literature.

Yet, there are two issues to address before making a firm conclusion. The first regards the power of our statistical analysis; the second relates to the failed terrorist attacks in July 21. As for the first concern, our very short run analysis has only 237 observations (including pre-treatment observations). Detecting an effect is therefore difficult (power calculations show a power of .509 for our privacy rights index and 0.298 for the procedural rights index, below the conventional .8 level for inferring null effect, see Appendix $\mathrm{I}$ for more details). As a consequence, we have elected not to comment on the statistical significance of our very short run estimates. The reader may worry that the effect we find in the short run and medium run is due to increased number of observations as we are pooling more weeks together (the number of observations for the short run and medium run analyses are 405 and 436, respectively). To alleviate this concern, we reproduce the analysis on a week-by-week basis for the first six weeks after the attacks, where the number 
of observations is very similar across weekly estimates 13 Any difference regarding the treatment effect or the statistical significance of the point estimates can no longer be attributed to larger sample or differential power.

Figure 3 displays the coefficients and 95\% confidence intervals for weeks 1 to 6 after the attacks for both privacy rights (left panel) and procedural rights (right panel). The results are very consistent with our more aggregated analysis. They indicate that the delay we observe is not due to weekly outliers. There is a progressive increase in the support for restrictions of liberties up to week four after the attacks, followed by a stabilization (for privacy rights) or a slight decrease (for procedural rights).

Figure 3: Treatment effects for Privacy and Procedural Rights weekly
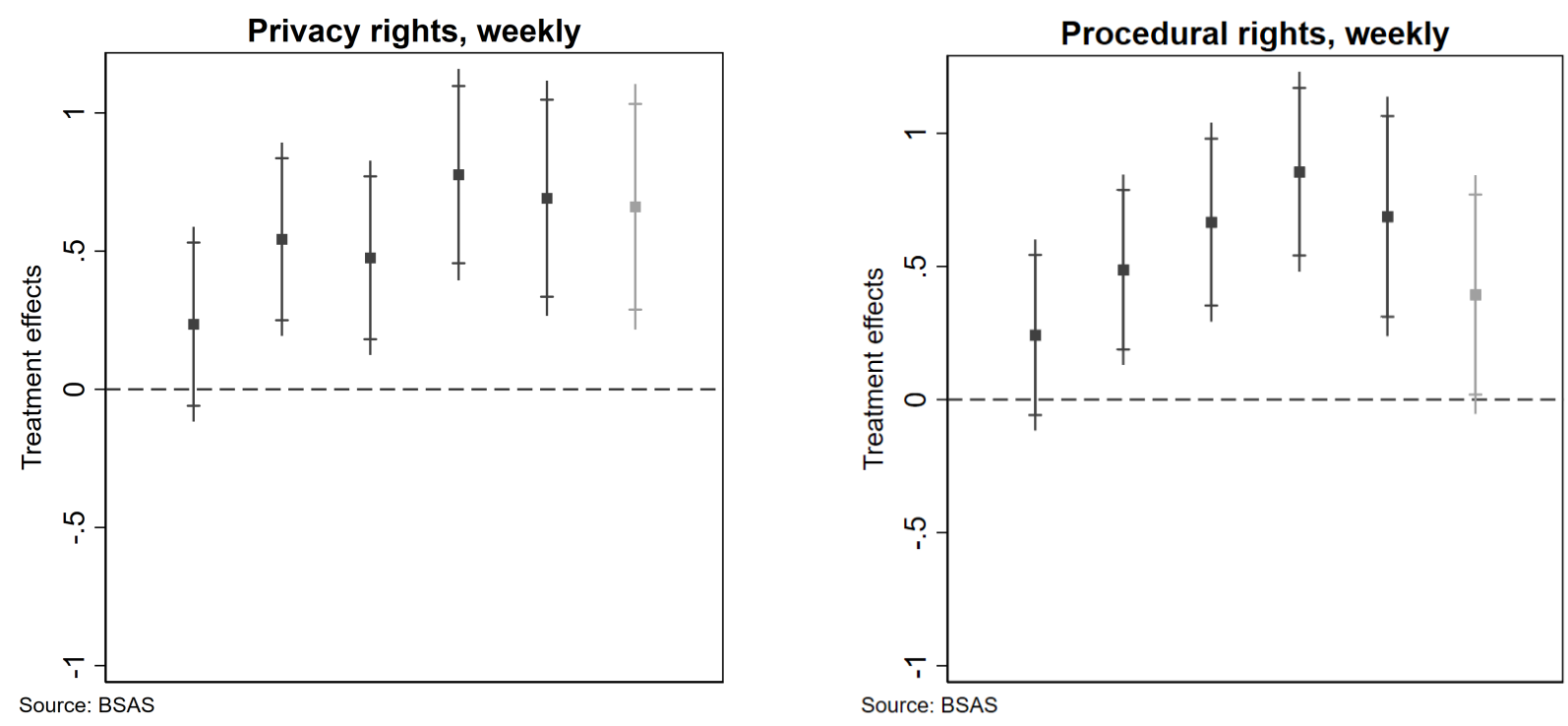

Notes. For more information regarding the analysis, see Figure 2 above.

As for the failed terrorist attacks on the 21st of July, our weekly analysis reveals that the estimates in week two (from $15 / 7$ to $21 / 7$ ) is not statistically different than the estimates in week three $(22 / 7$ to $28 / 7)$ for both privacy rights (p-value of 0.678 ) and procedural rights (p-value of 0.360). In Appendix J, we conduct our full aggregate analysis across our three time periods and find null effect throughout. While we cannot definitely argue that the failed attacks had no effect, it is very unlikely they are driving our results.

\footnotetext{
${ }^{13}$ The number of observations in weekly regressions for privacy rights are 224 for the second week, 216 for the third week, 199 for the fourth, 181 for the fifth and 172 for the sixth week. For procedural rights, the number of observations is 227 for the second week, 223 for the third, 205 for the fourth, 189 for the fifth, 180 for the sixth week. There is not enough observations after week 6 to carry out a weekly analysis.
} 


\subsection{Comparison with other survey items}

We explore the effect of the London bombings on other survey items included in the $B S A S$. For these issues the attacks are arguably more likely to trigger emotional responses. We monitor respondents' opinions on whether international human rights laws impede effective military operations, and their attitudes towards heightened security measures, namely whether harsh provisions should target all UK residents or only foreign nationals. We use these questions as proxies for aggressiveness and out-group-hostility respectively. We then examine whether the bombings changed people's selfidentification using a survey item that asks respondents how they identify themselves: British, English, Welsh, Northern Irish, etc. We refer to the "British identity" as a proxy for people's sense of broad belonging, and the "English identity" to denote a narrow definition of self. This "identity test" follows Depetris-Chauvin et al. (2018), with the only difference that we look at national and sub-national ties rather than ethnic vs national self-identification ${ }^{14}$ Finally, we use survey items that focus on respondents' levels of trust towards other people in society and towards the government (see Appendix C for the wording of the survey items).

We also look at the impact of the bombings on respondents' assessment of risks, namely their evaluation of the likelihood of another terrorist attacks in the UK. We further use a question on the salience of terrorism, that is whether the threat of terrorism is of great concern for the respondents. The former is meant to capture the effect of new information, the latter is harder to interpret, though the literature usually relates it to perception of threat (Huddy et al. 2005) .

The evolution of public opinion in the very short, short, and medium runs on these survey items differ significantly from the dynamics of attitudes on privacy and procedural rights. On the permissiveness of aggressive policy interventions, feeling English, generalized trust, and political trust, we see an immediate increase followed by a decrease (the coefficient for feeling British being 0.043 in the very short run and 0.05 in the short run). The volatility of public opinion on these dimensions conforms with the findings in the literature of emotional responses to terrorism, which emphasizes the role played by individuals' feelings and defense mechanisms in coping with security threats in general and terrorism in particular (Huddy et al. 2005). When it comes to risk assessment, we observe an immediate increase consistent with previous survey findings (Rubin et al. 2005; 2007). This feeling of higher threat remains stable across the time periods we consider, suggesting that

\footnotetext{
${ }^{14}$ We prefer the broad/narrow distinction to a regional/national one since English identity can refer both to the region, but also to a certain sense of nationality (e.g., England competes in many sport tournaments separately from other sub-national units of the United Kingdom).
} 
the attack played the role of an information shock. In other items, there appears to be no reaction at all.

The analysis of this section suggests terrorist attacks do not seem to trigger an immediate change in attitudes on curtailments of civil freedoms. Rather, the public reacts with a delay to the terrorist shock when it comes to privacy and procedural rights. This opens the possibility for indirect effects, driven by elite persuasion (a question we investigate in Section 5). Further, our results point to the absence of an emotional and irrational response regarding restrictive security policies (unlike other relevant dimension displayed in Figure 4). The higher level of fear of terrorism does not come along with demands for counter-terrorist measures that infringe upon core individual freedoms. If anything, it goes in the opposite direction. As such, our findings point to a public that tends to distinguish between emotions and policy responses, adopting a rather "wait and see" attitude for the latter. In the next section, we explore who drives the dynamics uncovered above. 
Figure 4: Related issues: Threat, ingroup Bias, SELF-IDENTIFICATion And trust

(a) Likelihood and salience of threat

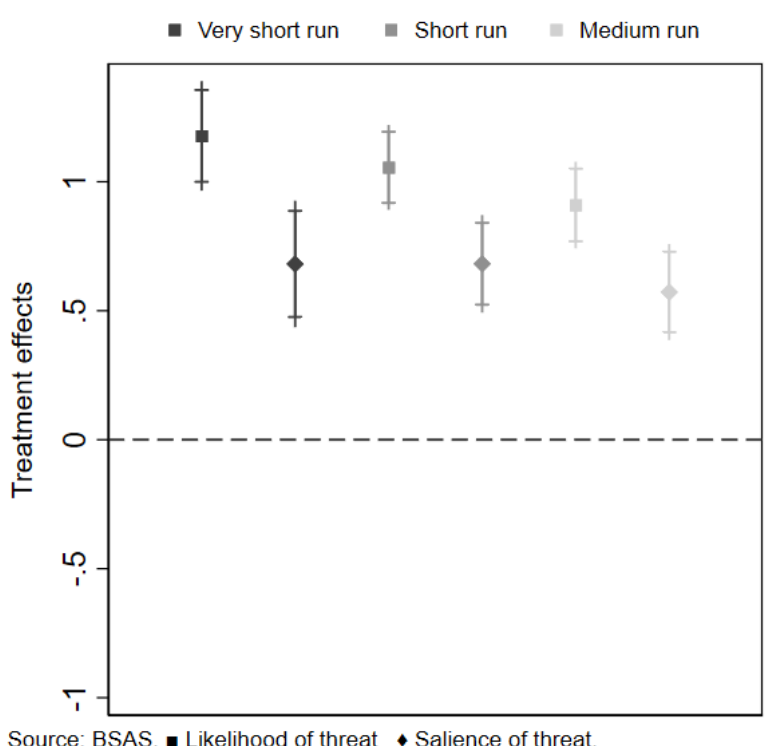

(c) Self-identification

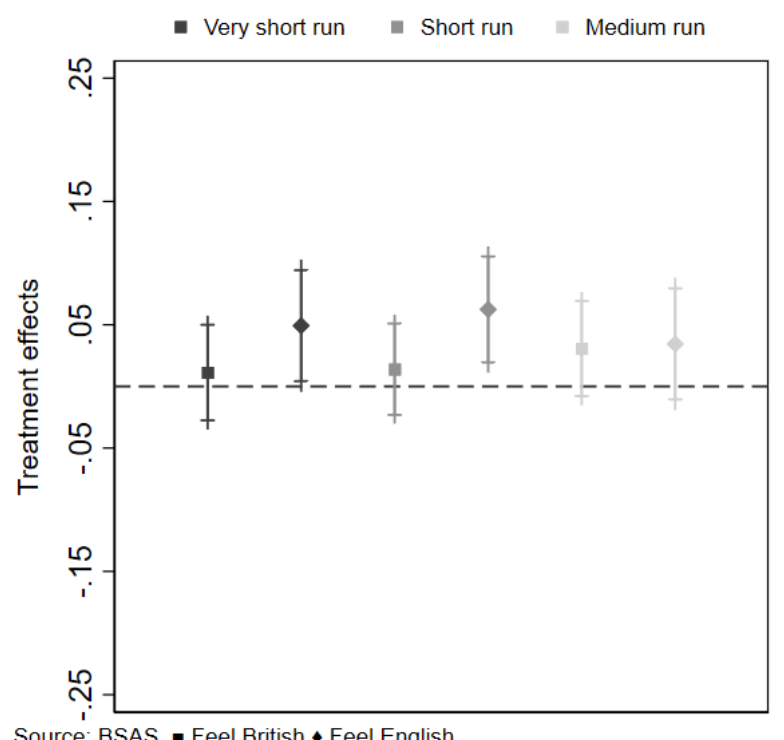

(b) Aggressiveness and in-group bias

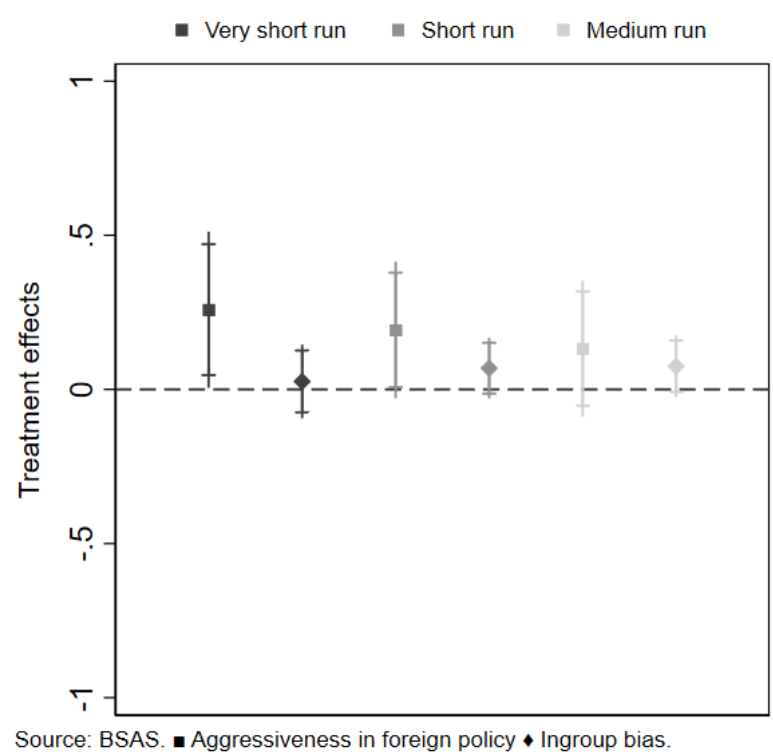

(d) Generalized and political trust

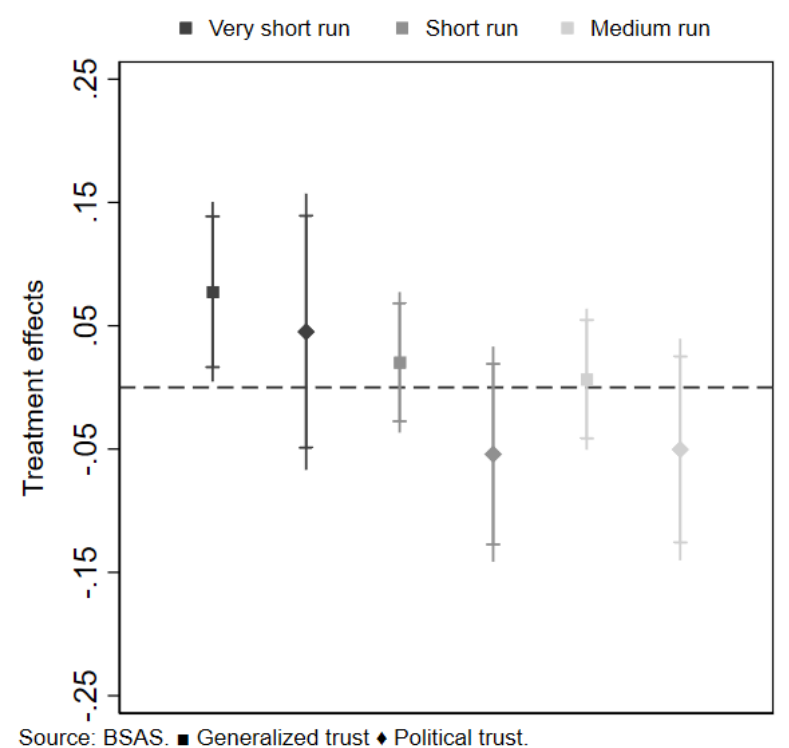

Notes. For more information regarding the analysis, see Figure 2 above. 


\section{An investigation of possible mechanisms}

In this section, we investigate two possible sources of influence: politicians and the media. These two channels are not perfectly distinguishable. Politicians may affect the public through the media and that the media influence politicians' discourse. Yet, we do our best to tell them apart. Further, the empirical analysis in this section does not claim to uncover causal effects. Our tests are not definitive. Rather, they are exploratory analyses indicative of some probable (or improbable) sources of influence.

To test for the first mechanism, one would like to look at the effect of a new counter-terrorist policy proposal on public attitudes towards security measures keeping all other factors constant. Our data do not allow us to perform such a test. What we can do is to check whether changes in public opinion occur in the aftermath of a widely publicized speech on counter-terrorism delivered by the y the incumbent PM, Tony Blair, in a press conference in August 5, 2005.

We replicate our empirical strategy, using the speech as treatment (the results are displayed in Figure K.1 in Appendix K). Our findings suggest the public is not affected by Tony Blair's endorsement of harsh counter-terrorist policies. Attitudes on both privacy and procedural rights did not change after the speech.

However, there are reasons not to read too much into this test. First, the Blair's speech came at a later point in time when respondents could have been already influenced by media coverage or previous public interventions by Tony Blair or prominent politicians. Second, our "treated group" spans several weeks (due to low number of observations) increasing the risk of finding no effect as other events may influence respondents during the "treatment" period.

To complement our analysis we look at how the treatment effect varies with respondents' partisan leaning. We distinguish between respondents who reported to have voted for Labour in the 2005 United Kingdom general election and voters of other parties (mostly respondents reporting to have voted for the Conservatives and a few for the Liberal Democrats). Before describing the result of this test, it should be noted that partisanship may be affected by the London bombings and this may induce post-treatment bias (such as party switches). Yet, our findings, displayed in Figure $5 \mathrm{a}$ for privacy rights and Figure $5 \mathrm{~b}$ for procedural rights, are in line with a large literature that attributes to left-wing voters a greater concern for the protection of individual freedoms than conservatives, with the latter giving priority to security (Davis, 2007; Davis and Silver, 2004; Huddy 
Figure 5: LABOUR AND NON-LABOUR VOTERS

(a) Privacy rights

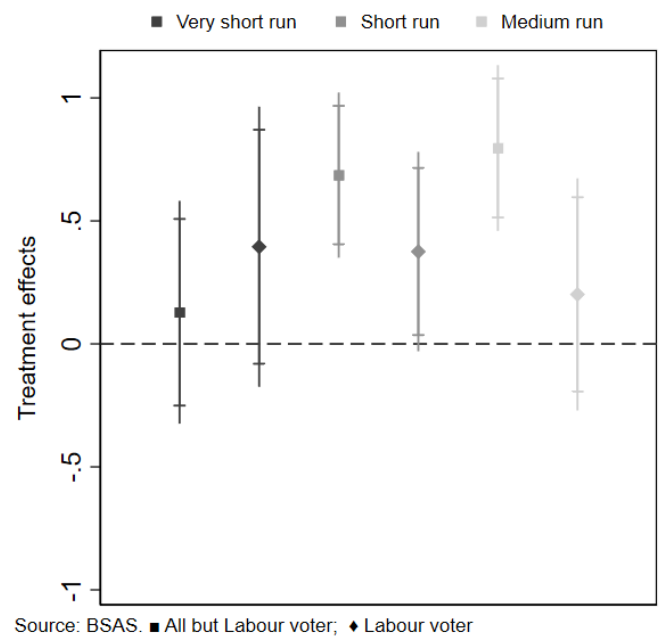

(b) Procedural rights

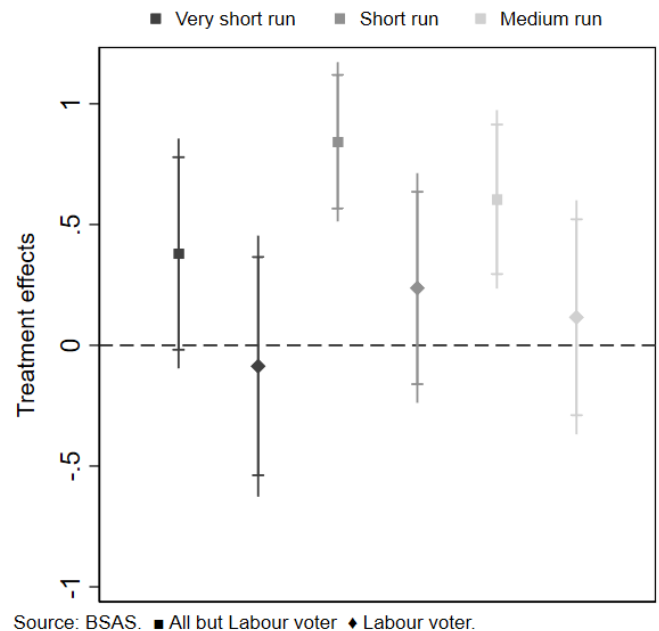

The sample for this analysis is splitted according to partisanship, distinguishing between Labour supporters and all others. The specification for every analysis is the same as that presented in Table 1.

et al., 2007). The dynamics we uncovered are due to non-Labour voters. Left-leaning respondents do not appear to respond to the terrorist shock in any of the time period considered.

The lack of reaction by Labour voters runs against the idea that politicians influence voters' stances on counter-terrorist policies. Building on Cukierman and Tommasi (1998) and Calvert (1985), if politicians lead public attitudes, with the Labour party in office, one should have observed the reverse effect with left-wing respondents reacting more than right-wing individuals.15 While we cannot offer a definitive conclusion, the evidence in favor of politicians' influence appears to be weak, at best.

Turning to the possible influence exercised by the media, ideally, we would have liked to compare the effect of $7 / 7$ on respondents with high exposure to media with those with low exposure. A way to operationalize this test would have been to interact the treatment effect with newspaper readership controlling for media coverage. Unfortunately, our data do not allow us to perform such analysis due to sample size of respondents reporting to read a newspaper and issues of post-

\footnotetext{
${ }^{15}$ Cukierman and Tommasi (1998) and Calvert (1985) both show that public attitude changes most when voters receive a piece of news from an unlikely source. In our case, we would expect the Conservative party to be on average more favorable to tougher measures than the Labor party. When Tony Blair endorses restrictions to privacy and procedural rights, this should signal to skeptical voters that these policies are necessary (just like it took a Nixon to convince the American voters that normalizing diplomatic relations with communist China was the right policy). Consequently, we should observe a bigger change in attitudes for Labour respondents than Conservative respondents if the hypothesis that attitudes change because of politicians' actions is correct. As the reverse holds true in our data, this suggests that the hypothesis is to be rejected.
} 
Figure 6: ARTICLES COUNT ON TERRORISM

(a) Total daily articles

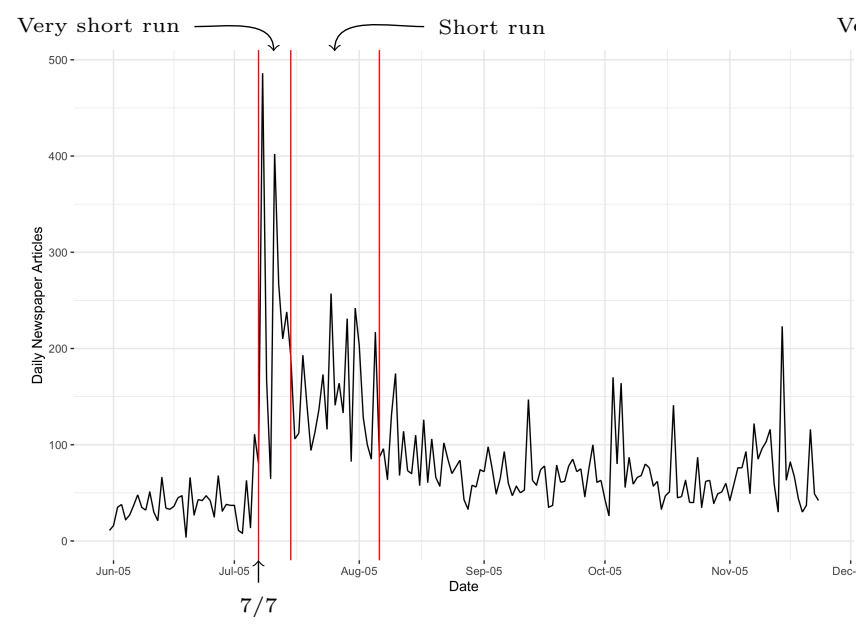

(b) Weekly articles by publication

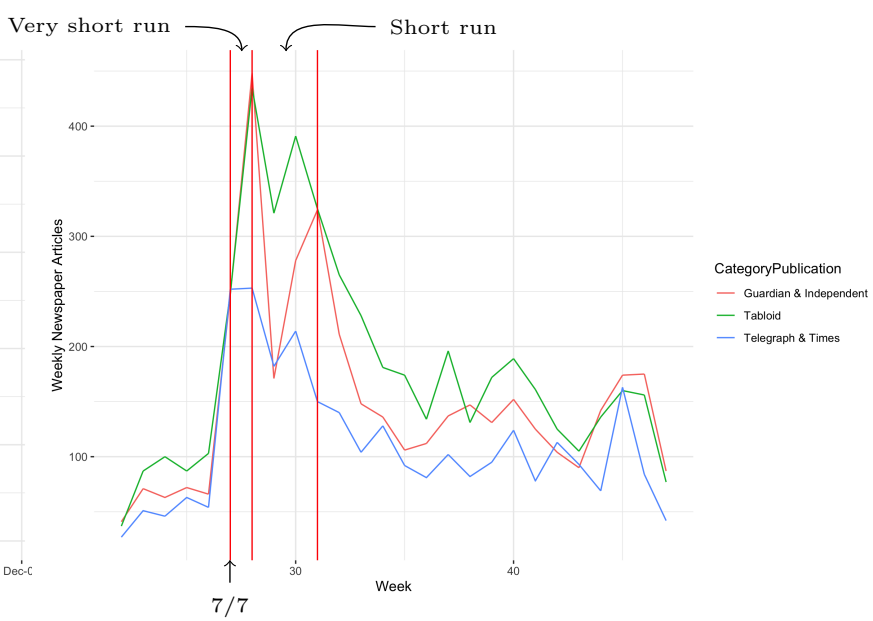

treatment bias. As a second best, we look at media coverage in the aftermath of the attack, without attempting to measure the effect of say coverage on respondents.

Figure 6 shows the number of articles on terrorism, both on a daily and weekly basis, for the whole period of the $B S A S$ interviews were carried out (for an overview of the methodology used in this figure and others, see Online Appendix $\mathrm{M}$. In both graphs, the first red line depicts the day of the London bombings $(7 / 7 / 2005)$ the second and third red lines demarcate the very short run (8/7 to $14 / 7)$ and the short run $(15 / 7$ to $6 / 8)$, respectively.

As Figure 6a shows, the daily number of newspaper articles mentioning terrorism picked on the day of the attack and the first week after the bombings. The frequency remained high in the month that followed showing that the issue maintained high salience for a significant period. While this is not surprising given the scale of the attacks, it shows that terrorism was in the mind of respondents interviewed in the very short and short runs ${ }^{16}$

Figure 6b displays the number of articles on terrorism on a weekly basis by publication type, with daily tabloids including The Sun, Daily Mail, Daily Mirror, Daily Star, Daily Express and their Sunday versions. Two patterns emerge from the figure. First, tabloids tend to publish more articles on terrorism than their more prestigious counterparts. This effect is partly mechanical due to the number of outlets considered (though, tabloids tend to report less political news). Second, tabloids exhibit a peak in the number of articles right in the middle of our short run period.

To make inference more robust, we also look at the content of newspaper articles in Figure 7. Again, we observe variations in mentions that appear to match the dynamics of public opinion. In

\footnotetext{
${ }^{16}$ It should be noted that the number of daily articles on terrorism does not change dramatically on the day after the failed attacks, reinforcing our view that this event is not at the origin of our findings.
} 
the very-short run, we see little references to terror laws, counter-terrorism, or references to policy measures such as anti-terrorist laws or counter-terrorism. These themes become more prominent in the short-run (Figures $7 \mathrm{a}$ and $7 \mathrm{~b}$. Inversely, mentions of freedom are higher in the very short run rather than in the short run when they reach their minimum (Figure $7 \mathrm{~d}$ ). The pattern for security is different due to the G8 meeting taking place on 6-8 July 2005, which dominated the news before the terrorist attacks. ${ }^{17}$ We see much more variation in the middle run (due to the longer period considered). In particular, the content of articles tend to match the political debates, with, for example, mentions of "freedom," "terror laws," and "counterterrorism" all exhibiting a spike around the discussion of the new Terrorism Act proposed in September 2005 (the introduction of this bill probably explains as well the late spike in the number of daily articles in Figure 6a).

Additional analysis in Online Appendix $\mathrm{M}$ provides further evidence that media reporting and public opinion changes seem to move in parallel. We show that articles mentioning "solidarity" peaking just after the attack (potentially explaining why generalized trust increases in the very short run, see Figure 4d), whereas references to "Ban", "Detain and Torture", "Intelligence", "Muslims" are all higher in the short run than in the first week after the attack. Finally, we see no clear pattern in mentions of "Terrorism Act" until October/November, that is the time the bill was introduced in the Parliament.

Figure 8 plots the log odds ratio of words appearing in articles published in the very short run (the first week) as compared to articles published in the short run (the first month). 18 Term frequencies are calculated separately within the very short and short term subsets of the population of articles. We then compute the log ratio of the most frequent fifty words in the first week to the most frequent words in the first month. The top fifteen rows are words that appear with greater frequency in the press in the first week relative to the words in the first month, while the bottom fifteen rows show words appearing more frequently in the first month relative to the first week.

The very short run is dense of words describing the London bombings. The first two upper rows of Figure 8 indicate that "train" and "bus" are the most frequent terms in the first week from the bombings, followed by words that can be related to a description of the events taking place on the day of the attack (e.g. "London", "people", "terrorist", "day", "tube", "bombs"). In contrast, in the short run, words related to the perpetrators of the attacks ("bombers", "muslim") and their

\footnotetext{
${ }^{17}$ The references to freedom in the weeks prior to the attack are likely to be due to the debate around the Identity Cards Bill introduced by the government into the Commons on 25 May. This conjecture is supported by an analysis of mentions of 'ID cards' displayed in Figure M.2b in Online Appendix M.

${ }^{18}$ See Wasow (2020) for an application of this approach to the case of violent/peaceful protests.
} 
Figure 7: WEEKLY ARTICLES ON TERRORISM AND COUNTERTERRORISM.

(a) Terror laws
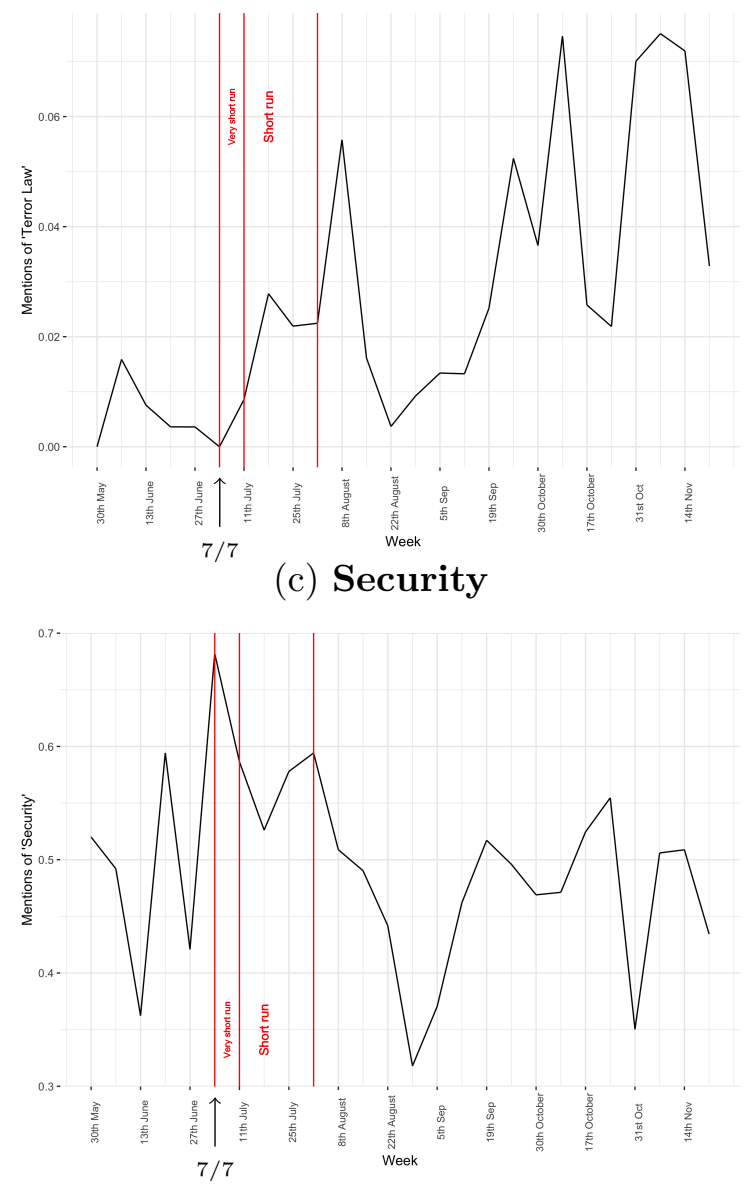

(b) Counterterrorism

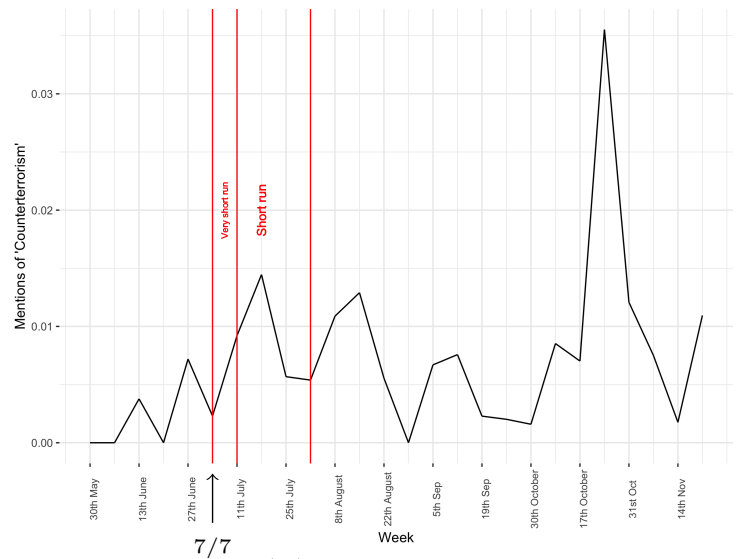

(d) Freedom

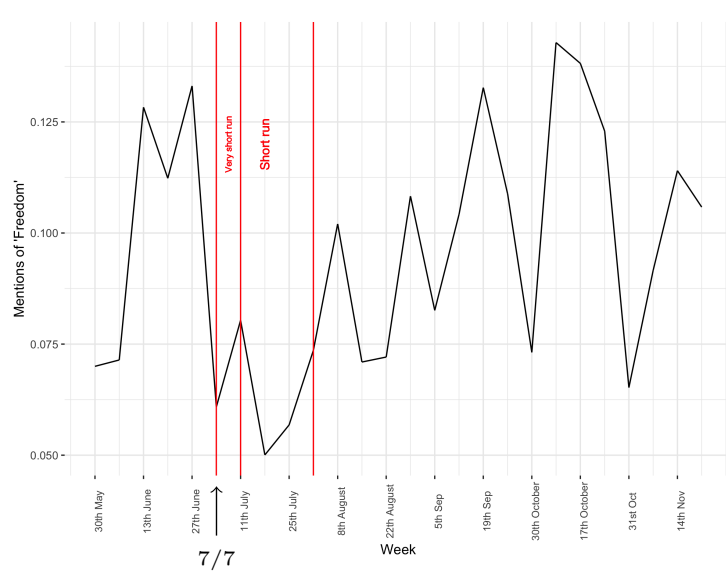




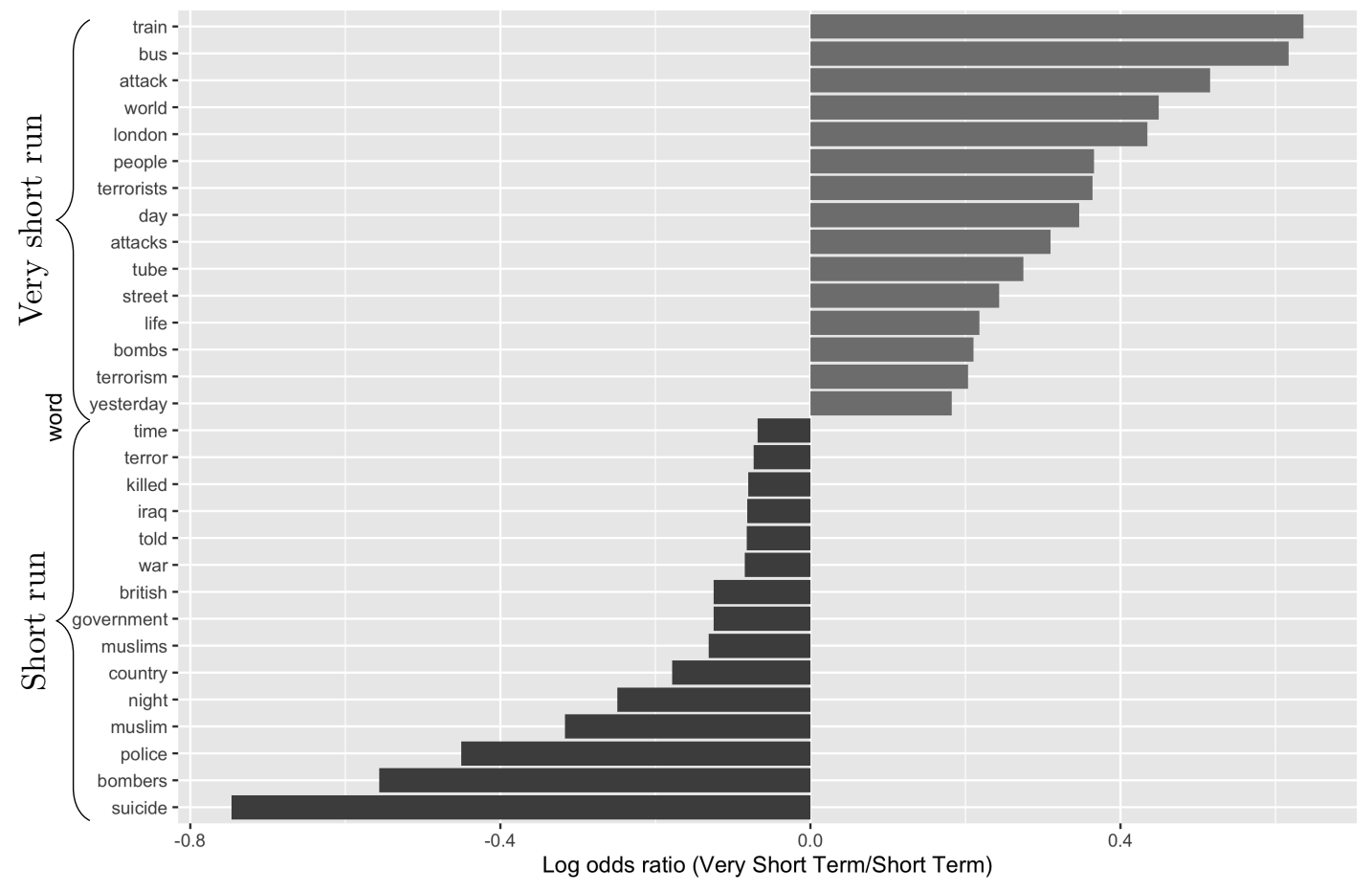

consequences ("police", "government", "war", "told") become much more frequent than in the very short run. A word cloud analysis in Appendix $M$ of the most used terms in the first week and the first month from the attacks displays a similar pattern (Figure M.4).

We further look at the log odd ratios of words recurring in tabloids and non-tabloids in the very short run and the short run. As discussed above, the number of articles devoted to terrorism by tabloids exhibits a peak right in the middle of the short run period - a peak we do not observe for other types of outlets. Figure $9 \mathrm{a}$ and Figure $9 \mathrm{~b}$ shows these odd ratios. Compared to the first week after the attack, we observe that words such as "muslim/s" and "bombers/suicide" prevail in the short run in both tabloid and non-tabloids. This points to similar patterns of profiling, with an emphasis on the religious connotation of the perpetrators of the bombings. What is more striking is the difference in the "institutions" mentioned. For non tabloids, both the government and the police are highly recurring in the short run, whereas the only institution appearing in tabloids is the police. A number of articles in tabloids urged the implementation of tighter counter-terrorist laws "similar to the US's Patriot Act"19 and were rather critical of the government response to the attacks, perceived as too slow 20 Of course, these are simply descriptions of the media discourse

\footnotetext{
${ }^{19}$ The Express, 23 July 2005. "Evil men behind failed bombs will be quaking in their boots: Analysis".

${ }^{20}$ The Express, 25 July 2005. "Anti-terror law can't wait for MPs' holidays".
} 
but they seem to suggest a difference in framing between outlet types, with tabloids proposing a narrative independent of politicians.

Tabloids in the UK, with the exception of the Daily Mirror (Yougov, 2017), tend to lean towards the right of the ideological spectrum. This is consistent with the heterogeneous effects displayed in Figure5 where we observed in the short and medium runs a change in attitudes in favor of restrictive measures solely driven by non-labor respondents. The readership of tabloids is also usually associated to low educated people and working classes (Newspaper Marketing Agency, 2008). Our data allows distinguishing respondents according to whether they hold (or do not hold) a university degree. The analysis shows a weak effect of the bombings for both privacy and procedural rights in the very short run for both college and non-college educated respondents. Changes in attitudes towards more restrictive privacy and procedural rights occur in the short and medium run only for for less educated respondents (results are displayed in FigureL.1a and Figure L.1b in the Appendix L).

None of the evidence presented above are definitive. They seem to indicate that the change in emphasis is related to the dynamics of public attitudes on security. While we cannot exclude that media reporting simply responds to the public mood, at least, our findings are consistent with media persuasion, which has been documented in other settings such as Brooks and Manza (2013) and Merolla and Zechmeister (2009) in experiments, Cho et al.(2003) in the aftermath of 9/11 in the USA, and Solheim (2019) in the case of the 2015 Charlie Hebdo and Hyper Cacher attacks. Our results potentially highlight the role of more popular media, the tabloids, in shaping public opinion. This last finding may be an interest avenue for future research. 
Figure 9: RATIO OF TERM FREQUENCIES IN THE VERY SHORT RUN VERSUS THE SHORT RUN IN TABLOIDS AND NON-TABLOIDS

(a) Tabloids

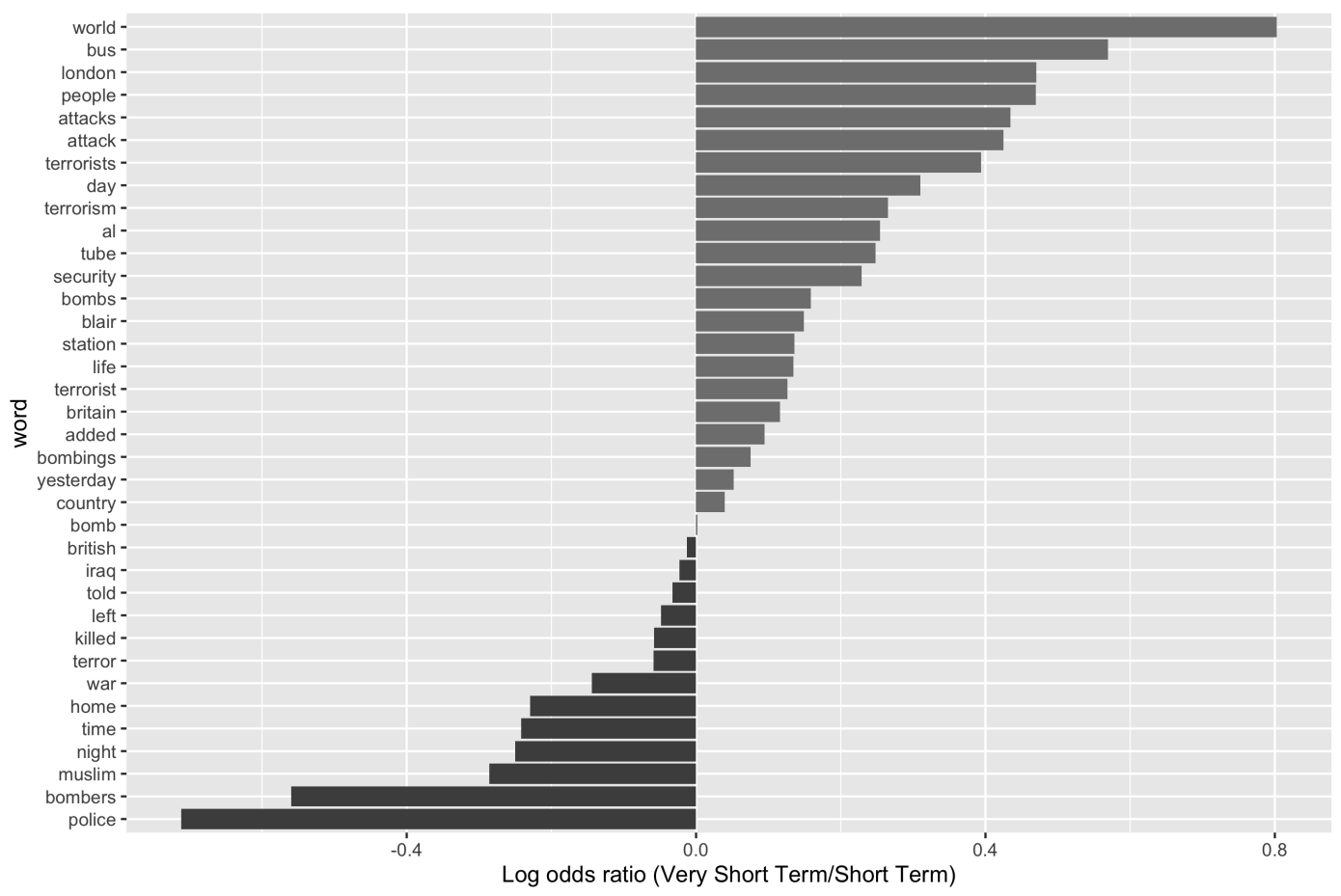

(b) Non-Tabloids

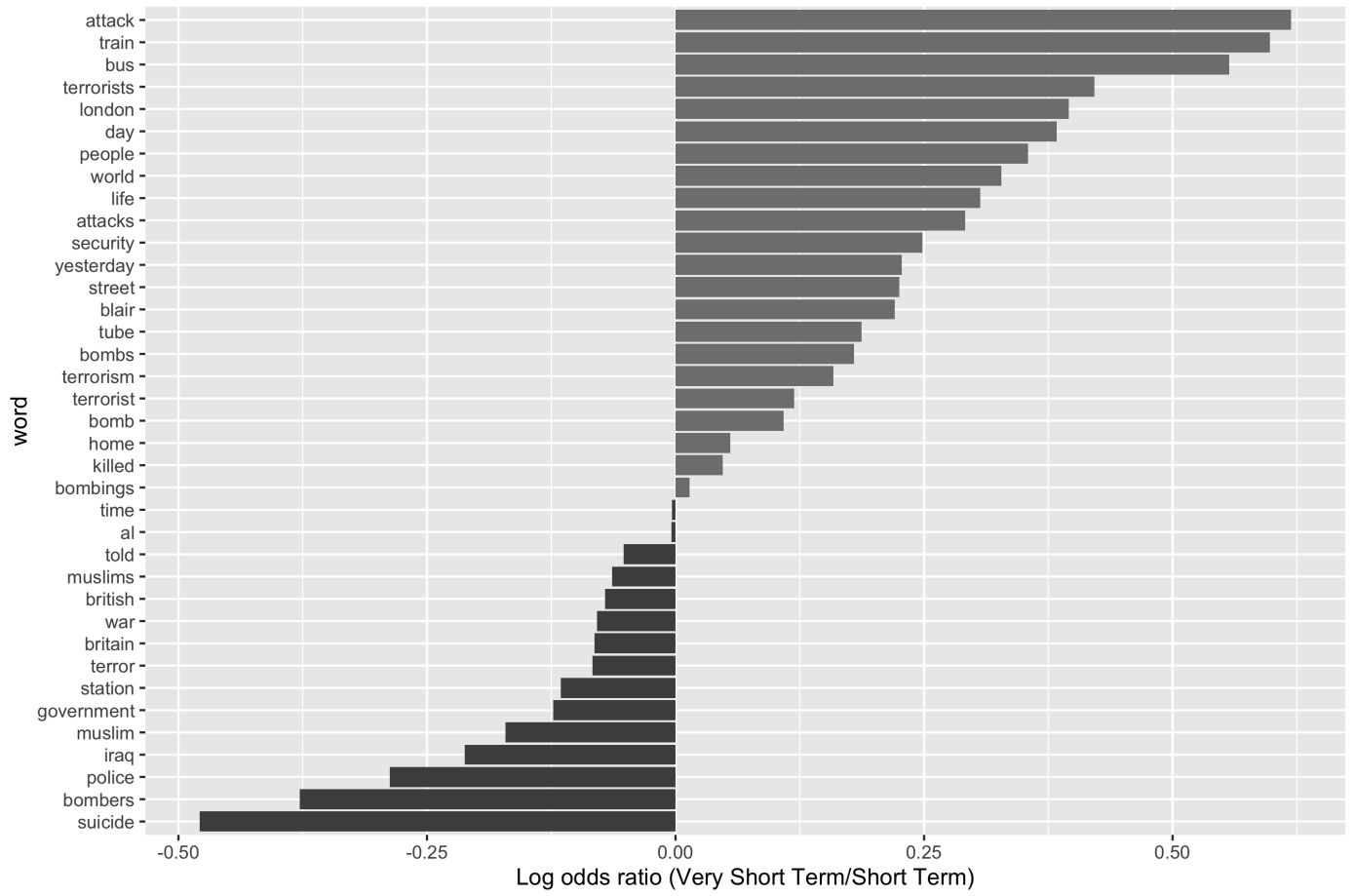

Tabloids consist of The Sun, Daily Mail, Daily Mirror, Daily Star, Daily Express and their Sunday versions. Non tabloids are The Times of London, The Telegraph, The Guardian/Observer, Independent. 


\section{Conclusion}

When and how do people change opinion on civil liberties after a terrorist attack? How much of the reversal of attitudes on individual rights is due to public fear? What is the role of elites, such as politicians and the media, in shaping the demands for security measures? Our analysis of dynamics of public stances on privacy and procedural rights after the $7 / 7$ bombings in London suggest these possible answers. Citizens demand more security after a terrorist event, but with a delay. Support for restrictions on core freedom is rather low in the very first week, and increases only in the short run and medium runs. These dynamics point to a "reasoned" response to the attack, with individuals waiting for more information on the nature of the threat before changing their opinion on fundamental liberties. Ancillary tests suggest a limited role played by political elites in shaping the stances on the trade-off between liberty and security and indicate the media as one source of persuasion, with the mainstream press (tabloids) possibly more influential than more prestigious outlets (non-tabloids).

We believe the implications of these findings extend beyond the specific shock we study. Recent research (Breton and Eady, 2021; Nussio, 2020) show that major terrorist attacks still trigger an emotional response on non-policy dimensions. These reactions have been used by politicians and pundits to justify changes in policies. The issues at stake may have changed, they regard immigration restrictions rather than security measures, but political elites still do not appear to let a crisis go to waste. If our research is any indication, media persuasion may be a driving force behind changes (or lack thereof) in support for closing borders. We hope that our causal results and more descriptive findings can help researchers understand how Western democracies continue to adopt anti-liberal policies with public approval. 


\section{References}

Alexander, David A., and Susan Klein. 2005. "The psychological aspects of terrorism: from denial to hyperbole." Journal of the Royal Society of Medicine 98 (12): 557-562.

Arvanitidis, Paschalis, Athina Economou, and Christos Kollias. 2016. "Terrorism's effects on social capital in European countries." Public Choice, 169 (3-4): 231-250.

Balcells, Laia, and Gerard Torrats-Espinosa. 2018. "Using a natural experiment to estimate the electoral consequences of terrorist attacks." Proceedings of the National Academy of Sciences, 115 (42): 10624-10629.

Blackwell, Matthew, Stefano Iacus, Gary King, and Giuseppe Porro. 2009. "cem: Coarsened exact matching in Stata" The Stata Journal, 9 (4): 524-546.

Böhmelt, Tobias, Vincenzo Bove, and Enzo Nussio. 2020. "Can terrorism abroad influence migration attitudes at home?." American Journal of Political Science, 64 (3): 437-451.

Boomgaarden, Hajo G., and Claes H. de Vreese. 2007. "Dramatic real-world events and public opinion dynamics: Media coverage and its impact on public reactions to an assassination." International Journal of Public Opinion Research, 19 (7): 354-366.

Boydstun, Amber E., Jessica T. Feezell, and Rebecca A. Glazier. 2018. "In the wake of a terrorist attack, do Americans' attitudes toward Muslims decline?." Research $\&$ Politics, 5(4).

Bozzoli, Carlos, and Cathérine Müller. 2011. "Perceptions and attitudes following a terrorist shock: Evidence from the UK." European Journal of Political Economy, 27: S89-S106.

Breton, Charles, and Gregory Eady. 2021. "Does International Terrorism affect Public Attitudes toward Refugees? Evidence from a Large-scale Natural Experiment." Forthcoming in Journal of Politics. Available here.

Brooks Clem and Jeff Manza. 2013. Whose Rights?: Counterterrorism and the dark side of American public opinion. New York: Russel Sage Foundation.

Bux, Shahid M., and Sarah M. Coyne. 2009. "The Effects of Terrorism: The Aftermath of the London Terror Attacks." Journal of Applied Social Psychology, 39 (12): 2936-2966.

Crenshaw, Martha. 1986. "The psychology of political terrorism". Political Psychology, 21(2): 379413.

Calvert, Randall L. 1985. "The value of biased information: A rational choice model of political advice." The Journal of Politics, 47 (2): 530-555.

Castanho Silva, Bruno. 2018. "The (non) impact of the 2015 Paris terrorist attacks on political attitudes." Personality and Social Psychology Bulletin, 44(6): 838-850.

Cho Jaeho, Michael P. Boyle, Heejo Keum, Mark D. Shevy, Douglas M. McLeod, Dhavan V. Shah, and Zhongdang Pan. 2013. "Media, terrorism, and emotionality: Emotional differences in media content and public reactions to the September 11th terrorist attacks". Journal of Broadcasting \& Electronic Media, 47(3): 309-327. 
Cukierman, Alex, and Mariano Tommasi. 1998. "When does it take a Nixon to go to China?" American Economic Review, 88 (1): 180-197.

Davis Darren W. and Brian D. Silver. 2004. "Civil liberties vs. security: Public opinion in the context of the terrorist attacks on America". American Journal of Political Science, 48(1): 28-46.

Davis Darren W. 2007. Negative liberty: Public opinion and the terrorist attacks on America. New York: Russell Sage Foundation Publications.

Depetris-Chauvin, Emilio, Ruben Durante, and Filipe Campante. 2020. "Building nations through shared experiences: Evidence from African football." American Economic Review, 110 (5): 15721602 .

Dinesen, Peter Thisted, and Mads Meier Jæger. 2013. "The effect of terror on institutional trust: New evidence from the 3/11 Madrid terrorist attack." Political Psychology 34 (6): 917-926.

Elsayed AEA and de Grip, Andries. 2013. "Terrorism and integration of Muslim immigrants". No. 014. Maastricht University, Research Centre for Education and the Labour Market (ROA).

Echebarria-Echabe, Agustin, and Emilia Fernández-Guede. 2006. "Effects of terrorism on attitudes and ideological orientation." European Journal of Social Psychology, 36 (2): 259-265.

Economou, Athina, and Christos Kollias. 2019. "Security policy preferences of EU citizens: Do terrorist events affect them?." Public Choice, 178 (3): 445-471.

Epifanio, Mariaelisa. 2011. "Legislative response to international terrorism." Journal of Peace Research, 48 (3): 399-411.

Finseraas, Henning, Niklas Jakobsson, and Andreas Kotsadam. 2011. "Did the Murder of Theo van Gogh Change Europeans' Immigration Policy Preferences?." Kyklos, 64 (3): 396-409.

Finseraas, Henning, and Ola Listhaug. 2013. "It can happen here: The impact of the Mumbai terror attacks on public opinion in Western Europe." Public Choice, 156 (1-2): 213-228.

Gautier, Pieter A., Arjen Siegmann, and Aico Van Vuuren. 2009. "Terrorism and attitudes towards minorities: The effect of the Theo van Gogh murder on house prices in Amsterdam." Journal of Urban Economics, 65 (2): 113-126.

Getmansky, Anna, and Thomas Zeitzoff. 2014. "Terrorism and voting: The effect of rocket threat on voting in Israeli elections." American Political Science Review, 108(3): 588-604.

Geys, Benny, and Salmai Qari. 2017. "Will you still trust me tomorrow? The causal effect of terrorism on social trust." Public Choice, 173 (3-4): 289-305.

Giani, Marco. 2020. "Fear without Prejudice in the Shadow of Jihadist Threat". Comparative Political Studies, 54 (6): 1058-1085.

Giani, Marco, and Luca Merlino. 2021. "Terrorist attacks and minority perceived discrimination". British Journal of Sociology, 72 (2): 286-299.

Giner-Sorolla, Roger, and Angela T. Maitner. 2013. "Angry at the unjust, scared of the powerful: Emotional responses to terrorist threat." Personality and Social Psychology Bulletin, 39 (8): 10691082. 
Gross, Kimberly, Paul R. Brewer, and Sean Aday. 2009. "Confidence in government and emotional responses to terrorism after September 11, 2001." American Politics Research, 37 (1): 107-128.

Hainmueller, Jens. 2012. "Entropy balancing for causal effects: A multivariate reweighting method to produce balanced samples in observational studies." Political analysis, 30: 25-46.

Hanes, Emma, and Stephen Machin. 2014. "Hate crime in the wake of terror attacks: Evidence from 7/7 and 9/11." Journal of Contemporary Criminal Justice, 30 (3): 247-267.

Huddy Leonie, Stanley Feldman, Charles Taber and Gallya Lahav. 2005. "Threat, anxiety, and support of antiterrorism policies". American Journal of Political Science, 49(3): 593-608.

Iacus, Stefano M., Gary King, and Giuseppe Porro. 2012. "Causal inference without balance checking: Coarsened exact matching." Political analysis, 20 (1): 1-24.

Jakobsson, Niklas, and Svein Blom. 2014. "Did the 2011 terror attacks in Norway change citizens' attitudes toward immigrants?." International Journal of Public Opinion Research, 26 (4): 475-486.

Joslyn, Mark R., and Donald P. Haider-Markel. 2007, "Sociotropic concerns and support for counterterrorism policies." Social Science Quarterly, 88 (2): 306-319.

Joslyn, Mark R., and Donald P. Haider-Markel. 2018. "The direct and moderating effects of mass shooting anxiety on political and policy attitudes." Research $\&$ Politics, 5 (3): 1-9.

Kam, Cindy D., and Jennifer M. Ramos. 2008. "Joining and leaving the rally: Understanding the surge and decline in presidential approval following 9/11." Public Opinion Quarterly, 72(4): 619-650.

Legewie, Joscha. 2013. "Terrorist events and attitudes toward immigrants: A natural experiment." American Journal of Sociology, 118 (5): 1199-1245.

Lerner, Jennifer S., Roxana M. Gonzalez, Deborah A. Small, and Baruch Fischhoff. 2003. "Effects of fear and anger on perceived risks of terrorism: A national field experiment." Psychological Science, 14 (2): 144-150.

Metcalfe, Robert, Nattavudh Powdthavee, and Paul Dolan. 2011. "Destruction and distress: using a quasi-experiment to show the effects of the September 11 attacks on mental well-being in the United Kingdom." The Economic Journal, 121 (550): F81-F103.

Merolla, Jennifer L., and Elizabeth J. Zechmeister. 2009. Democracy at risk: How terrorist threats affect the public. University of Chicago Press.

Merolla, Jennifer L., and Elizabeth J. Zechmeister. 2013. "Evaluating political leaders in times of terror and economic threat: The conditioning influence of politician partisanship." The Journal of Politics, 75 (3): 599-612.

Montalvo, Jose G. 2011. "Voting after the bombings: A natural experiment on the effect of terrorist attacks on democratic elections." Review of Economics and Statistics 93 (4): 1146-1154.

Mueller, John, and Mark G. Stewart. 2018. Public opinion and counterterrorism policy. Washington, DC: Cato Institute. 
Muñoz, Jordi, Albert Falcó-Gimeno, and Enrique Hernández. 2019. "Unexpected event during survey design: promise and pitfalls for causal inference." Political Analysis, 28 (2): 186-206.

Nägel, Christof, and Mark Lutter. 2020. "The Christmas Market Attack in Berlin and Attitudes Toward Refugees: A Natural Experiment with Data from the European Social Survey." European Journal for Security Research 5 (2): 199-221.

Neumayer, Eric, Thomas Plumper, and Mariaelisa Epifanio. 2014. "The "Peer-Effect" in Counterterrorist Policies." International Organization (2014): 211-234.

NMA Newspaper Marketing Agency. Jan-Jun 2009.

Nussio, Enzo, Vincenzo Bove, and Bridget Steele. 2019. "The consequences of terrorism on migration attitudes across Europe." Political Geography, 75: 102047.

Nussio, Enzo. 2020. "Attitudinal and Emotional Consequences of Islamist Terrorism. Evidence from the Berlin Attack." Political Psychology, 41 (6): 1151-1171.

Park Allison, John Curtice, Katarina Thomson, Miranda Phillips and Mark Johnson. 2007. British Social Attitudes Survey: British Social Attitudes: The 23rd report. SAGE Publications

Perrin, Andrew J., and Sondra J. Smolek. 2009. "Who trusts? Race, gender, and the September 11 rally effect among young adults." Social Science Research, 38 (1): 134-145.

Perry, Ronald W., and Michael K. Lindell. 2003. "Understanding citizen response to disasters with implications for terrorism." Journal of Contingencies and Crisis Management, 11 (2): 49-60.

Prager, Fynnwin, Garrett Ryan Beeler Asay, Bumsoo Lee, and Detlof von Winterfeldt. 2011. "Exploring Reductions in London Underground Passenger Journeys Following the July 2005 Bombings." Risk Analysis, 31 (5): 773-86.

Pudles, Daniel. 2007. "The real price of freedom". The Economist.

Rogers, M. Brooke, Richard Amlôt, G,. James Rubin, Simon Wessely, and Kristian Krieger.2007. "Mediating the social and psychological impacts of terrorist attacks: The role of risk perception and risk communication." International Review of Psychiatry, 19(3): 279-288.

Rubin, G. James, Chris R. Brewin, Neil Greenberg, John Simpson, and Simon Wessely. 2005. "Psychological and Behavioural Reactions to the Bombings in London on 7 July 2005: Cross Sectional Survey of a Representative Sample of Londoners." BMJ 331 (7517): 606.

Rubin, G. James, Chris R. Brewin, Neil Greenberg, Jamie Hacker Hughes, John Simpson, and Simon Wessely. 2007. "Enduring Consequences of Terrorism: 7-Month Follow-up Survey of Reactions to the Bombings in London on 7 July 2005." The British Journal of Psychiatry, 190 (4): 350-56.

Sheppard, Ben, James G. Rubin, Jamie K. Wardman, and Simon Wessely. 2006. "Terrorism and dispelling the myth of a panic prone public". Journal of Public Health Policy, 27: 219-245.

Solheim, Øyvind Bugge. 2019. "Are we all Charlie? How media priming and framing affect immigration policy preferences after terrorist attacks." West European Politics (2019): 1-25.

Sunstein, Cass R. 2003. "Terrorism and probability neglect" Journal of Risk and Uncertainty, 26 (2-3): 121-136. 
Van de Vyver, Julie, Diane M. Houston, Dominic Abrams, and Milica Vasiljevic. 2016. "Boosting Belligerence: How the July 7, 2005, London Bombings Affected Liberals' Moral Foundations and Prejudice." Psychological Science, 27 (2): 169-77.

Van Hauwaert, Steven M., and Robert A. Huber. 2020. "In-group solidarity or out-group hostility in response to terrorism in France? Evidence from a regression discontinuity design." European Journal of Political Research, 59 (4): 936-953.

Viscusi, W. Kip, and Richard J. Zeckhauser. 2003. "Sacrificing civil liberties to reduce terrorism risks." Journal of Risk and Uncertainty, 26 (2-3): 99-120.

Wasow, Omar. 2020. "Agenda Seeding: How 1960s Black Protests Moved Elites, Public Opinion and Voting." American Political Science Review: 1-22.

Willer, Robb, and Nick Adams. 2008. "The threat of terrorism and support for the 2008 presidential candidates: Results of a national field experiment." Current Research in Social Psychology, 14 (1): $1-22$.

YouGov. How left or right-wing are the UK's newspapers? March 07, 2017. 


\section{Online Appendix \\ (Not for publication)}

\section{Table of Contents}

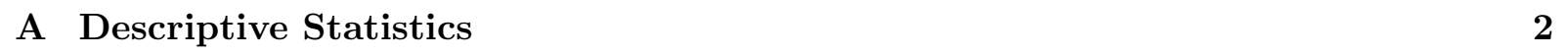

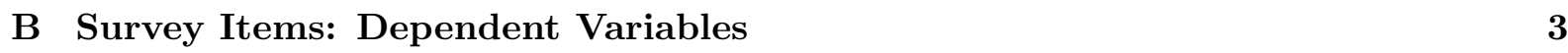

\begin{tabular}{|l|l|}
\hline C Survey Items: other dependent variables & 4
\end{tabular}

\begin{tabular}{|l|l}
\hline P Principal component analysis & 5
\end{tabular}

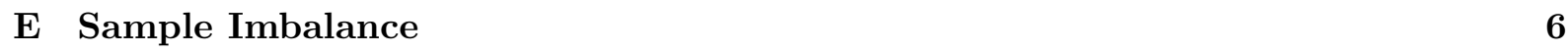

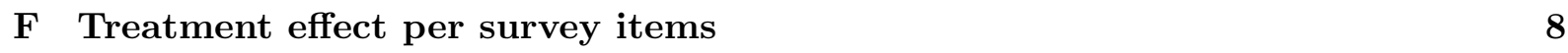

\begin{tabular}{lll}
\hline G & Further Robustness checks & 12
\end{tabular}

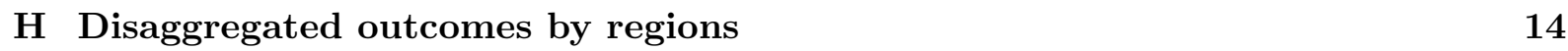

\begin{tabular}{lll}
\hline I Power calculations across time-intervals & 15
\end{tabular}

\begin{tabular}{|lr}
\hline J $\quad$ Failed attacks, 21 July 2005 & 16
\end{tabular}

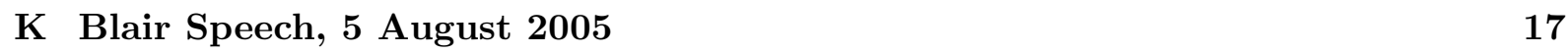

\begin{tabular}{llr}
\hline L & Heterogeneous effects: Education & 18
\end{tabular}

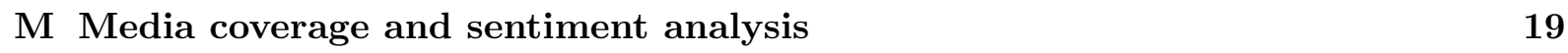




\section{A Descriptive Statistics}

\begin{tabular}{lcccccc|cc}
\hline & \multicolumn{7}{c}{ Control group } & \multicolumn{7}{c}{ Treatment group } & & \\
\hline & N.obs & Mean & St.dev & N.obs & Mean & St.dev & Min & Max \\
\cline { 2 - 8 } Privacy right & 149 & -.44 & 1.34 & 827 & .79 & 1.27 & -3.56 & 2.46 \\
Compulsory ID & 163 & 2.78 & 1.16 & 864 & 3.08 & 1.05 & 1 & 4 \\
Control email/phone & 163 & 3.05 & .91 & 867 & 3.28 & .84 & 1 & 4 \\
Ban free speech & 161 & 2.37 & 1.03 & 862 & 2.69 & 1.03 & 1 & 4 \\
Ban protest & 158 & 2.01 & 1.05 & 865 & 2.16 & 1.02 & 1 & 4 \\
Procedural right & 155 & -.37 & 1.44 & 821 & .70 & 1.43 & -3.60 & 2.48 \\
Arbitrary detention & 165 & 2.97 & 1.07 & 869 & 3.13 & .85 & 1 & 4 \\
Torture suspect & 164 & 1.47 & .83 & 865 & 1.76 & 1.02 & 1 & 4 \\
Tag suspect & 164 & 3.23 & .84 & 872 & 3.13 & .85 & 1 & 4 \\
Deny trial & 159 & 2.35 & 1.15 & 839 & 2.47 & 1.13 & 1 & 4 \\
\hline \hline Income & 189 & 7.92 & 4.8 & 902 & 9.25 & 5.02 & 1 & 17 \\
Education & 201 & 4.39 & 2.23 & 911 & 4.01 & 2.15 & 1 & 7 \\
Age & 201 & 53.33 & 18.14 & 911 & 49.23 & 17.80 & 18 & 99 \\
Gender & 201 & .58 & .49 & 911 & .60 & .49 & 0 & 1 \\
Children & 201 & .22 & .42 & 911 & .29 & .45 & 0 & 1 \\
Immigrant & 201 & .56 & .50 & 911 & .56 & .50 & 0 & 1 \\
Voted labour & 201 & .30 & .46 & 911 & .29 & .45 & 0 & 1 \\
\hline \hline
\end{tabular}

Table A.1: Descriptive statistics. 


\section{B Survey Items: Dependent Variables}

\section{Wording of survey items in the British Survey of Social Attitudes (UK, 2005) used to construct our dependent variables.}

- Privacy rights: "A number of measures have been suggested as ways of tackling the threat of terrorism in Britain. Some people oppose these because they think they reduce people's freedom too much. Others think that the reduction in freedom is a price worth paying. For each of the measures I mention, please say which of the views on this card comes closest to your own: (i) Following people suspected of involvement with terrorism, tapping their phones and opening their mail; (ii) having compulsory identity cards for all adults; (iii) Banning certain peaceful protests and demonstrations; (iv) banning certain people from saying whatever they want in public."

- Procedural rights: "A number of measures have been suggested as ways of tackling the threat of terrorism in Britain. Some people oppose these because they think they reduce people's freedom too much. Others think that the reduction in freedom is a price worth paying. For each of the measures I mention, please say which of the views on this card comes closest to your own: (i) Allowing the police to detain people for more than a week or so without charge if the police suspect them of involvement in terrorism; (ii) denying the right to a trial by jury to people charged with a terrorist-related crime; (iii) putting people suspected of involvement with terrorism under special rules, which would mean they could be electronically tagged, prevented from going to certain places, or prevented from leaving their homes at certain times; (iv) Torturing people held in British jails who are suspected of involvement in terrorism to get information from them, if this is the only way this information can be obtained." 


\section{Survey Items: other dependent variables}

Wording of survey items in the British Survey of Social Attitudes (UK, 2005) used as proxies of issues related to terrorist attacks.

- Likelihood of terrorist threat: "Please say whether you agree or disagree with each of the following statements. It is very likely that there will be a major terrorist attack in Britain in the next couple of years."

- Salience of terrorist threat: "Please say whether you agree or disagree with this statement. The threat of a terrorist attack in Britain is of great concern to me."

- Human rights: "Please say whether you agree or disagree with this statement. International human rights law prevents the armed forces from doing their job properly."

- In-group bias: "Please say whether you agree or disagree with this statement. Some people say that the same measures should apply to everyone in Britain, no matter where they are from. Others say stricter measures should apply to people who are from other countries."

- Self-identification: "Please say which, if any, of the words on this card describes the way you think of yourself. Please choose as many or as few as apply: (i) British; (ii) English; (iii) European; (iv) Irish; (v) Northern Irish; (vi) Scottish; (vii) Welsh; (viii) Other answer (write in); (ix) None of these."

- Generalized trust: "Generally speaking, would you say that most people can be trusted, or that you can't be too careful in dealing with people?"

- Political trust: "How much do you trust British governments of any party to place the needs of the nation above the interests of their own political party?" 


\section{Principal component analysis}

\begin{tabular}{|c|c|c|c|c|}
\hline & \multicolumn{4}{|c|}{ Score of first Component } \\
\hline & Compulsory ID & Control email/phone & Ban free speech & Ban protest \\
\hline Privacy right $t=0$ & .54 & .41 & .57 & .46 \\
\hline Privacy right $t=1$ & .51 & .49 & .52 & .51 \\
\hline & Arbitrary detention & Torture suspect & Tag suspect & Deny trial \\
\hline Procedural right $t=0$ & .53 & .41 & .54 & .51 \\
\hline Procedural right $t=1$ & .54 & .34 & .54 & .56 \\
\hline
\end{tabular}

Table D.1: PCA components. 


\section{E Sample Imbalance}

We are going to focus on the effect of terror attacks on public opinion in the very short run. Control and treated units may, in both cases, be unbalanced on socio-economic covariates. This would, in turn, jeopardize the validity of our results. We control for this potential bias in two ways.

- Entropy balancing. Our outcomes may therefore be biased if treated units were significantly less educated than control ones and therefore more likely to prioritize security over freedom. Following Hainmueller (2012), we weight control units such that the distribution of covariates among control units match the moment conditions (until skewness) of the treated units. After this pre-processing, covariate imbalance between control and treatment groups becomes negligible. We run again the main specification after weighting the data and, as shown in Table E.1, we find very similar results. Hence, sample imbalance does not seem to drive our outcomes.

- Coarsened exact matching. Following Iacus et al. (2012), we control for ex ante imbalance, by relying on non-parametric pretreatment matching using coarsened exact matching Blackwell et al., 2009), and ex-post, by adding controls to the OLS regression that estimates the (sample) treatment effect on the treated. Pre-treatment matching reveals that the UK control and treatment groups are unbalanced on education, age and whether the respondent has children living at home (age only). I match control and treated units exactly on education, while coarsening age in five years interval before matching them. Table E.1 shows that treatment effects after outliers' extraction based on coarsened exact matching stay similar in spite of a substantial drop in the effective sample. we can hence rule out that our results be driven by outliers in either the control or treatment group. 


\begin{tabular}{lccc|ccc}
\hline & \multicolumn{5}{c|}{ Privacy } & \multicolumn{3}{c}{ Procedural } \\
\cline { 2 - 7 } & Very short & Short & Medium & Very short & Short & Medium \\
\hline \multirow{5}{*}{ Treatment (0-1) } & .215 & $.582^{* * *}$ & $.613^{* * *}$ & .242 & $.684^{* * *}$ & $.440^{* * *}$ \\
Std Error & $(.174)$ & $(.133)$ & $(.138)$ & $(.182)$ & $(.135)$ & $(.148)$ \\
N.obs & 237 & 405 & 436 & 245 & 408 & 446 \\
\hline \multirow{7}{*}{ Treatment (0-1) } & .236 & $.591^{* * *}$ & $.613^{* * *}$ & .267 & $.670^{* * *}$ & $.525^{* * *}$ \\
Std Error & $(.179)$ & $(.135)$ & $(.138)$ & $(.178)$ & $(.142)$ & $(.141)$ \\
N.obs & 237 & 405 & 436 & 245 & 408 & 446 \\
\hline \multirow{7}{*}{ Treatment (0-1) } & .117 & $.521^{* * *}$ & $.483^{* * *}$ & .102 & $.564^{* * *}$ & $.333^{* *}$ \\
Std Error & $(.214)$ & $(.149)$ & $(.150)$ & $(.218)$ & $(.151)$ & $(.162)$ \\
N.obs & 180 & 299 & 306 & 189 & 308 & 316 \\
\hline
\end{tabular}

Table E.1: Treatment effects

(Full model, Entropy balancing, Coarsened exact matching) 


\section{F Treatment effect per survey items}

Table F.1: Public responses to terror attack: Privacy rights items

\begin{tabular}{|c|c|c|c|}
\hline & \multicolumn{3}{|c|}{ Compulsory ID } \\
\hline & Very Short Run & Short Run & Medium Run \\
\hline \multirow[t]{2}{*}{ Treatment } & 0.101 & $0.409^{* * *}$ & $0.375^{* * *}$ \\
\hline & $(0.145)$ & $(0.111)$ & $(0.110)$ \\
\hline Region FE & Yes & Yes & Yes \\
\hline N.obs & 254 & 427 & 456 \\
\hline \multirow[t]{3}{*}{ R-squared } & 0.12 & 0.09 & 0.10 \\
\hline & \multicolumn{3}{|c|}{ Tap email/phone } \\
\hline & Very Short Run & Short Run & Medium Run \\
\hline \multirow[t]{2}{*}{ Treatment } & $0.206^{*}$ & $0.244^{* * *}$ & $0.212^{* *}$ \\
\hline & $(0.112)$ & $(0.091)$ & $(0.089)$ \\
\hline Region FE & Yes & Yes & Yes \\
\hline N.obs & 254 & 424 & 458 \\
\hline \multirow[t]{3}{*}{ R-squared } & 0.09 & 0.05 & 0.08 \\
\hline & \multicolumn{3}{|c|}{ Ban protest } \\
\hline & Very Short Run & Short Run & Medium Run \\
\hline \multirow[t]{2}{*}{ Treatment } & 0.059 & $0.271^{* *}$ & $0.297^{* * *}$ \\
\hline & $(0.129)$ & $(0.106)$ & $(0.111)$ \\
\hline Region FE & Yes & Yes & Yes \\
\hline N.obs & 249 & 421 & 453 \\
\hline \multirow[t]{3}{*}{ R-squared } & 0.09 & 0.12 & 0.08 \\
\hline & \multicolumn{3}{|c|}{ Ban speech } \\
\hline & Very Short Run & Short Run & Medium Run \\
\hline Treatment & 0.152 & $0.334^{* * *}$ & $0.355^{* * *}$ \\
\hline & $(0.135)$ & $(0.110)$ & $(0.108)$ \\
\hline Region FE & Yes & Yes & Yes \\
\hline N.obs & 251 & 427 & 459 \\
\hline R-squared & 0.04 & 0.07 & 0.05 \\
\hline
\end{tabular}

${ }^{*} p<0.10,{ }^{* *} p<0.05,{ }^{* * *} p<0.01$ 
Table F.2: Public responses to terror attack: Procedural rights items

\begin{tabular}{|c|c|c|c|}
\hline & \multicolumn{3}{|c|}{ Stop and search } \\
\hline & Very Short Run & Short Run & Medium Run \\
\hline \multirow[t]{2}{*}{ Treatment } & 0.090 & $0.221^{* * *}$ & 0.063 \\
\hline & $(0.106)$ & $(0.081)$ & $(0.087)$ \\
\hline Region FE & Yes & Yes & Yes \\
\hline N.obs & 256 & 428 & 463 \\
\hline \multirow[t]{3}{*}{ R-squared } & 0.14 & 0.11 & 0.11 \\
\hline & \multicolumn{3}{|c|}{ Arbitrary detention } \\
\hline & Very Short Run & Short Run & Medium Run \\
\hline \multirow{2}{*}{ Treatment } & 0.199 & $0.411^{* * *}$ & $0.338^{* * *}$ \\
\hline & $(0.129)$ & $(0.097)$ & $(0.097)$ \\
\hline Region FE & Yes & Yes & Yes \\
\hline N.obs & 257 & 427 & 464 \\
\hline \multirow[t]{3}{*}{ R-squared } & 0.11 & 0.09 & 0.15 \\
\hline & \multicolumn{3}{|c|}{ Allow torture } \\
\hline & Very Short Run & Short Run & Medium Run \\
\hline \multirow[t]{2}{*}{ Treatment } & 0.101 & $0.516^{* * *}$ & $0.203^{* *}$ \\
\hline & $(0.107)$ & $(0.108)$ & $(0.100)$ \\
\hline Region FE & Yes & Yes & Yes \\
\hline N.obs & 254 & 423 & 461 \\
\hline \multirow[t]{3}{*}{ R-squared } & 0.11 & 0.11 & 0.06 \\
\hline & & Deny trial & \\
\hline & Very Short Run & Short Run & Medium Run \\
\hline \multirow[t]{2}{*}{ Treatment } & 0.130 & $0.283^{* *}$ & $0.214^{*}$ \\
\hline & $(0.142)$ & $(0.120)$ & $(0.121)$ \\
\hline Region FE & Yes & Yes & Yes \\
\hline N.obs & 250 & 417 & 452 \\
\hline R-squared & 0.10 & 0.09 & 0.06 \\
\hline
\end{tabular}

${ }^{*} p<0.10,{ }^{* *} p<0.05,{ }^{* * *} p<0.01$ 
Table F.3: Risk assessment, aggressiveness, in-group bias

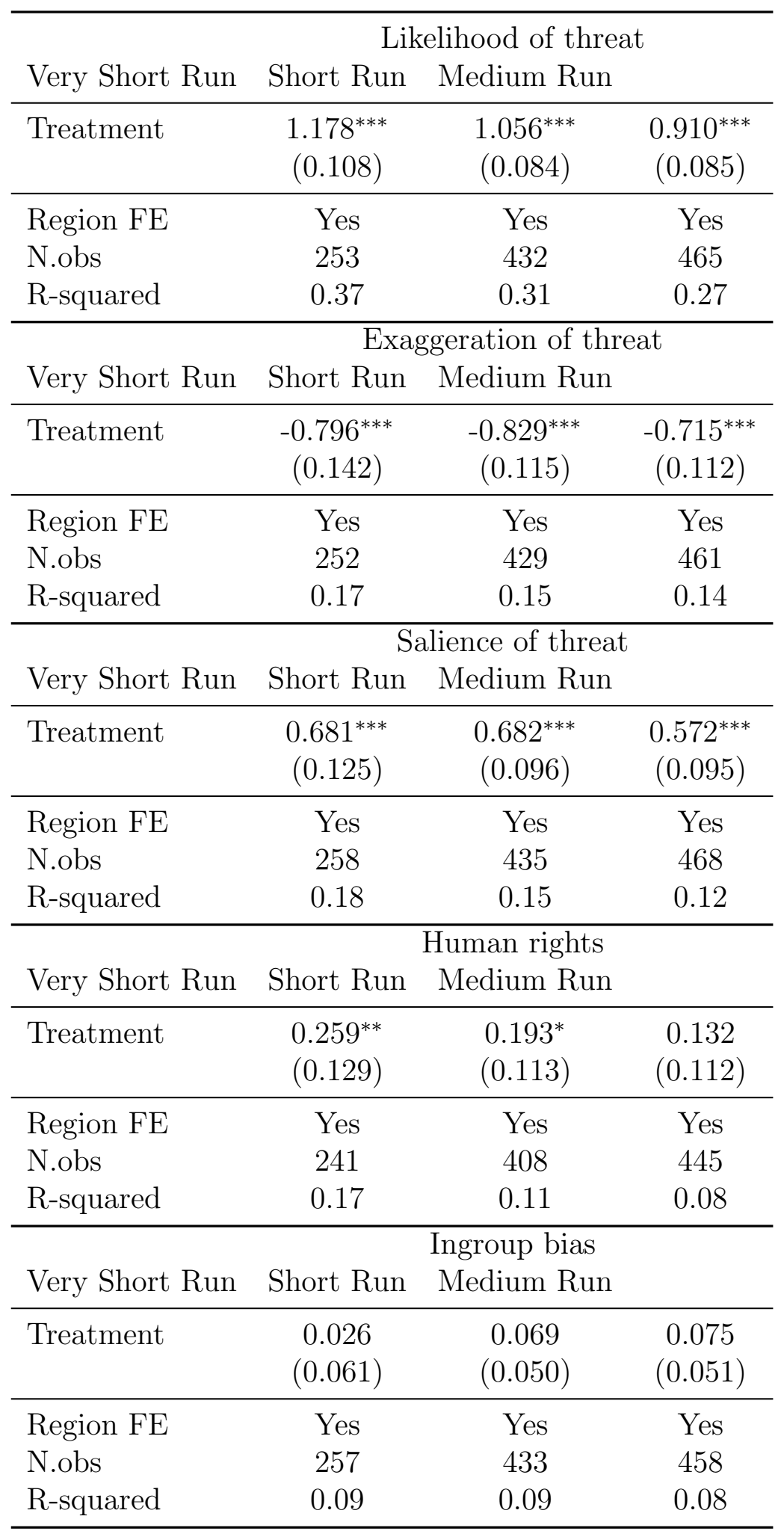

${ }^{*} p<0.10,{ }^{* *} p<0.05,{ }^{* * *} p<0.01$ 
Table F.4: Identity and trust

\begin{tabular}{|c|c|c|c|}
\hline Very Short Run & Short Run & $\begin{array}{l}\text { Feel English } \\
\text { Medium Run }\end{array}$ & \\
\hline Treatment & $\begin{array}{c}0.043 \\
(0.029)\end{array}$ & $\begin{array}{l}0.050^{* *} \\
(0.023)\end{array}$ & $\begin{array}{c}0.030 \\
(0.024)\end{array}$ \\
\hline Region FE & Yes & Yes & Yes \\
\hline N.obs & 1,010 & 1,751 & 1,945 \\
\hline R-squared & 0.19 & 0.18 & 0.14 \\
\hline Very Short Run & Short Run & $\begin{array}{l}\text { eeling British } \\
\text { Medium Run }\end{array}$ & \\
\hline Treatment & $\begin{array}{c}0.004 \\
(0.030)\end{array}$ & $\begin{array}{c}0.011 \\
(0.024)\end{array}$ & $\begin{array}{c}0.031 \\
(0.023)\end{array}$ \\
\hline Region FE & Yes & Yes & Yes \\
\hline N.obs & 1,010 & 1,751 & 1,945 \\
\hline R-squared & 0.05 & 0.06 & 0.03 \\
\hline Very Short Run & \multicolumn{3}{|c|}{ Generalized trust } \\
\hline Treatment & $\begin{array}{l}0.078^{* *} \\
(0.037)\end{array}$ & $\begin{array}{c}0.020 \\
(0.029)\end{array}$ & $\begin{array}{c}0.007 \\
(0.029)\end{array}$ \\
\hline Region FE & Yes & Yes & Yes \\
\hline N.obs & 727 & 1,296 & 1,415 \\
\hline R-squared & 0.09 & 0.09 & 0.07 \\
\hline Very Short Run & \multicolumn{3}{|c|}{ Political trust } \\
\hline Treatment & $\begin{array}{c}0.045 \\
(0.057)\end{array}$ & $\begin{array}{l}-0.054 \\
(0.044)\end{array}$ & $\begin{array}{l}-0.050 \\
(0.046)\end{array}$ \\
\hline Region FE & Yes & Yes & Yes \\
\hline N.obs & 728 & 1,287 & 1,408 \\
\hline R-squared & 0.08 & 0.07 & 0.07 \\
\hline
\end{tabular}




\section{G Further Robustness checks}

- Table G.1 provides the treatment effect for a slightly different specification, in which the principal component analysis used to construct our main dependent variables is estimated twice: once before the attack and once after it. As such, we wish to account for the fact that, as the variance of answers might have changed after the attack, factor scores should weight more single survey items on which polarization in answers increased. Treatment effects are, however, extremely close to those in our preferred specification (labeled Main Table).

- Table G.1 also provides treatment effects estimated with an ordered logit estimator, to account for the fact that non-linear models may better fit the data generating process. Results are substantially unchanged.

- Figure G.1 provides the same analysis as for Figure 2 displayed in the main body of the paper, but focuses on two placebo attitudes related to government spending. On the left panel, we use a survey item that refers to spending in healthcare ("What should the government choose between taxation and social services?"). On the right panel, we use a survey item that refers to spending for carer ("Should government pay carer benefits to let carers stop working?"). In both cases, for any interval of time, we obtain nonsignificant treatment effects.

\begin{tabular}{lccc|ccc}
\hline & \multicolumn{5}{c|}{ Privacy } & \multicolumn{3}{c}{ Procedural } \\
\cline { 2 - 7 } & Very short & Short & Medium & Very short & Short & Medium \\
\hline \multirow{7}{*}{ Treatment (0-1) } & .215 & $.582^{* * *}$ & $.613^{* * *}$ & .242 & $.684^{* * *}$ & $.440^{* * *}$ \\
Std Error & $(.174)$ & $(.133)$ & $(.138)$ & $(.182)$ & $(.135)$ & $(.148)$ \\
N.obs & 237 & 405 & 436 & 245 & 408 & 446 \\
\hline \multirow{7}{*}{ Alternative PCA assumptions } \\
Treatment (0-1) & .223 & $.571^{* * *}$ & $.594^{* * *}$ & .236 & $.668^{* * *}$ & $.424^{* * *}$ \\
Std Error & $(.174)$ & $(.131)$ & $(.134)$ & $(.180)$ & $(.140)$ & $(.147)$ \\
N.obs & 237 & 405 & 436 & 245 & 408 & 446 \\
\hline \multirow{7}{*}{ Ordered Logit estimator } \\
Treatment (0-1) & .255 & $.804^{* * *}$ & $.820^{* * *}$ & .234 & $.819^{* * *}$ & $.481^{* *}$ \\
Std Error & $(.233)$ & $(.195)$ & $(.196)$ & $(.231)$ & $(.191)$ & $(.186)$ \\
N.obs & 237 & 405 & 436 & 245 & 408 & 446 \\
\hline
\end{tabular}

Table G.1: Further robustness checks. 

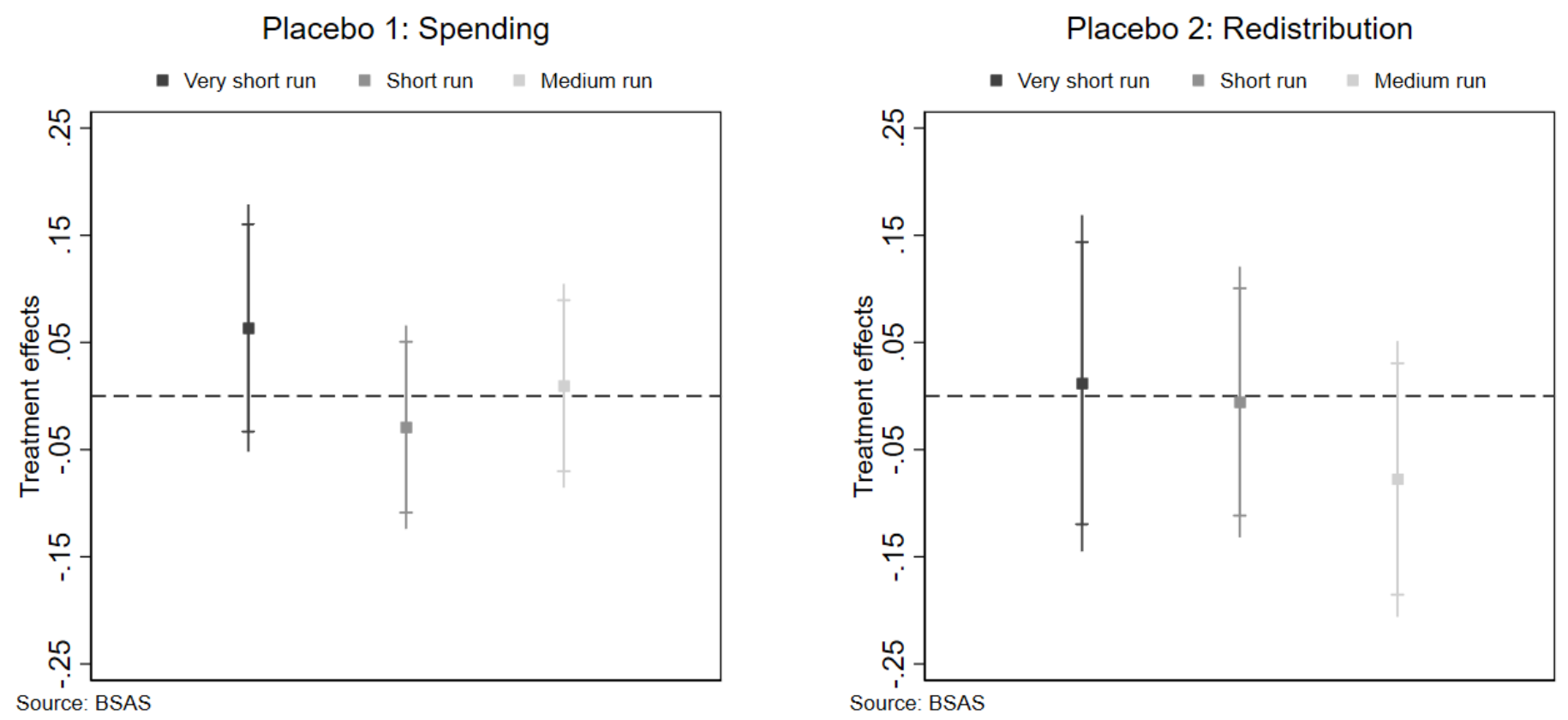

Figure G.1: Placebo issues 


\section{H Disaggregated outcomes by regions}

Table $\mathbf{H . 1}$ provides treatment effects by region for the whole interval of time. Most of our data come from England. Outcomes presented in Table H.1 must therefore be taken with care, as they are obtained from small samples.

\begin{tabular}{lcccc|cccc}
\hline & \multicolumn{4}{c|}{ Privacy } & \multicolumn{4}{c}{ Procedural } \\
\cline { 2 - 9 } & London & Rest of England & Wales & Scotland & London & Rest of England & Wales & Scotland \\
Treatment (0-1) & .389 & $.427^{* * *}$ & .448 & $1.083^{* * *}$ & $1.247^{*}$ & $.478^{* * *}$ & -.231 & .471 \\
Std Error & $(.519)$ & $(.141)$ & $(.791)$ & $(.339)$ & $(.631)$ & $(.150)$ & $(.826)$ & $(.382)$ \\
N.obs & 63 & 649 & 39 & 81 & 63 & 655 & 38 & 446 \\
\hline
\end{tabular}

Table H.1: Outcomes by region. 


\section{Power calculations across time-intervals}

One may wonder whether the lack of significance of our treatment effects in the very short run, coupled with its significance in the short-run, can be attributed to statistical power. Indeed, the effective sample increases for both the analysis of privacy and procedural rights from around 250 observations to around 400 when analysis the short run. However, it is unlikely that this difference be at the root of the attitudinal pattern discussed in the paper.

Indeed, Figure I.1 indicates that the statistical power to detect an effect corresponding to the point estimates in the very short run displayed in the paper is similar, and substantially below the standard 0.8 cutoff, to the one we would have had the effective sample in the very short run and short run been the same. For each subfigure of Figure I.1, the vertical regular (dashed) line marks the effective sample in the very short (short) run. The corresponding value of statistical power is similar despite the difference in effective sample.
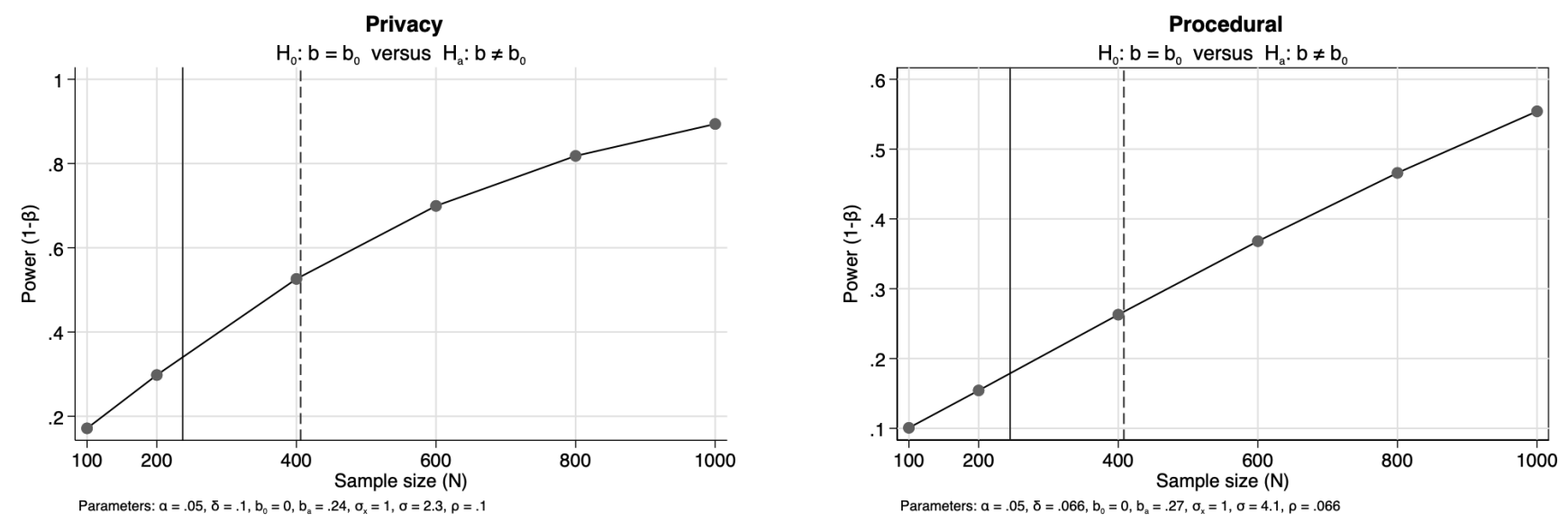

Figure I.1: Power calculations 


\section{J Failed attacks, 21 July 2005}
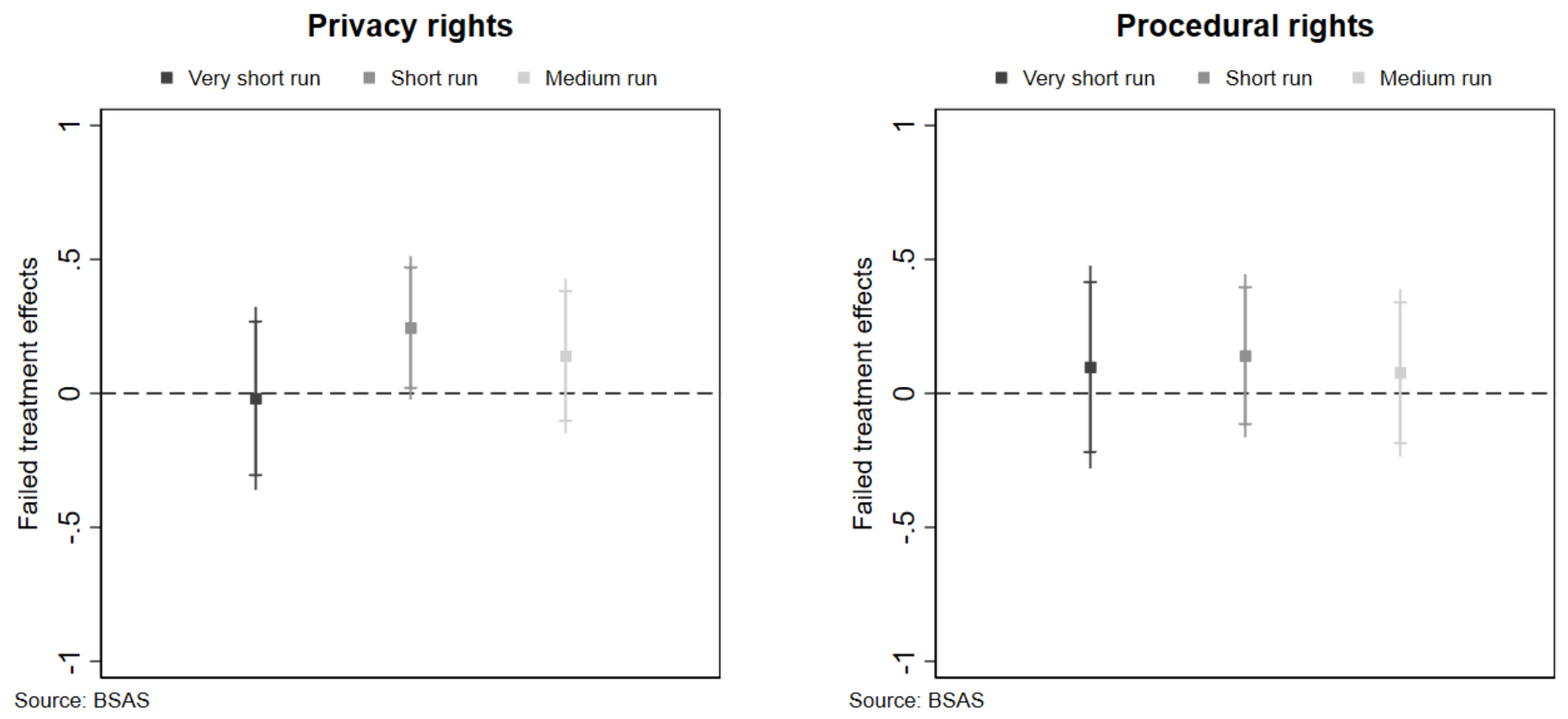

Figure J.1: Failed attacks, 21 July 2005 


\section{K Blair Speech, 5 August 2005}
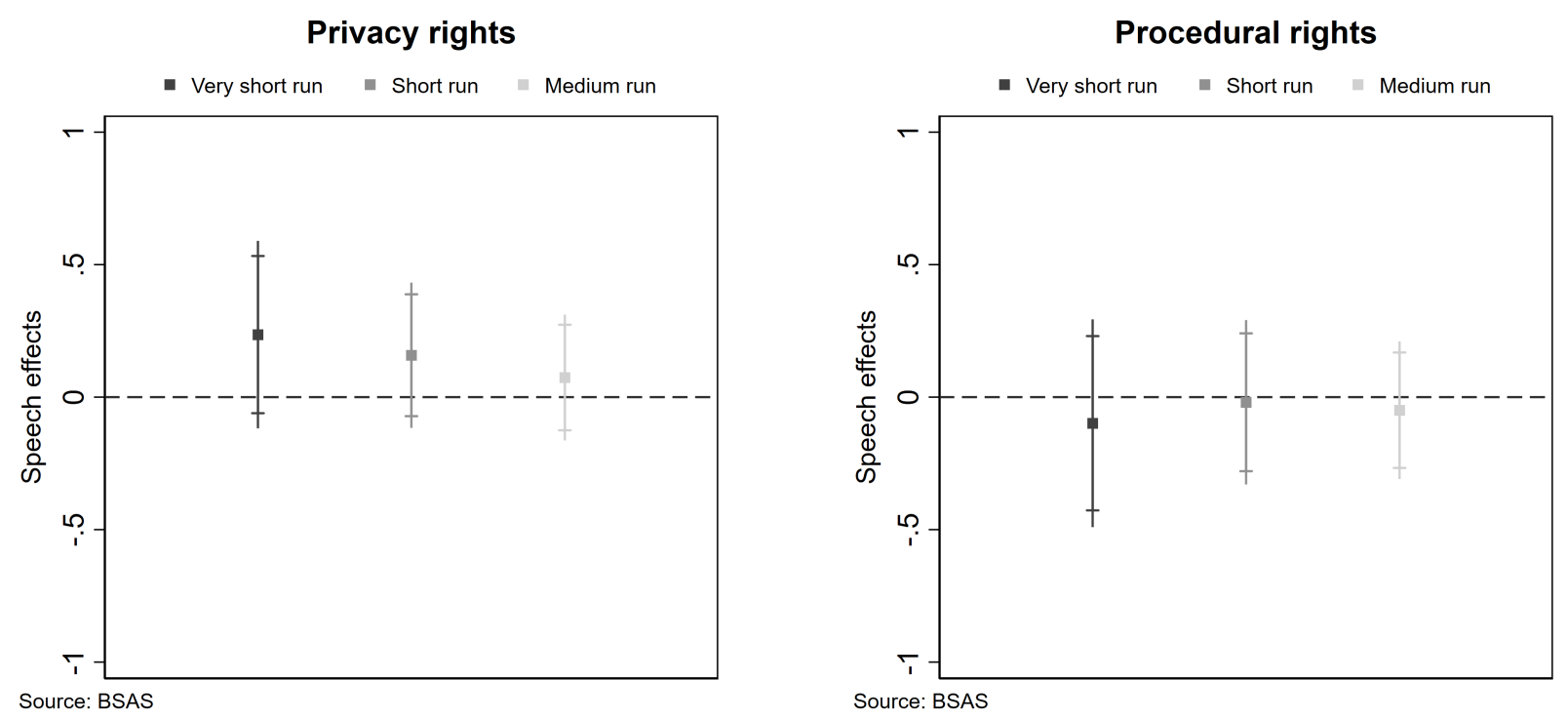

Figure K.1: Tony Blair Speech, 5 August 2005 


\section{Heterogeneous effects: Education}

Figure L.1: Heterogenous effects: Education

(a) Privacy rights: Non-graduate and graduate

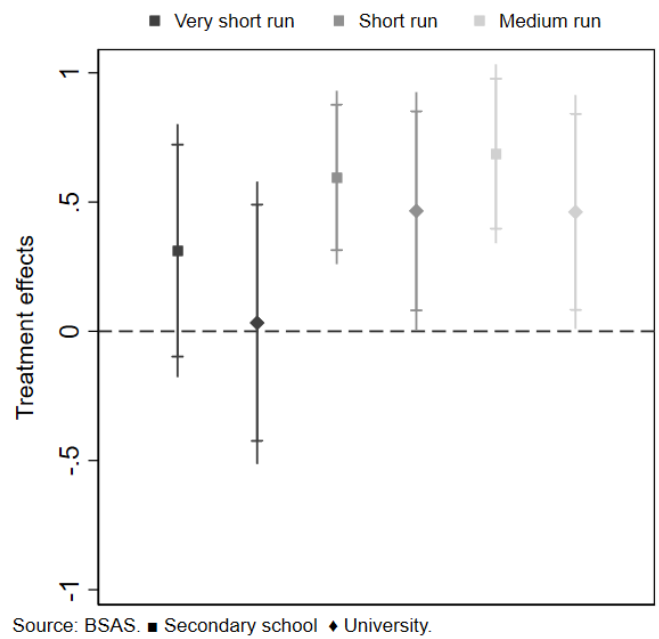

(b) Procedural rights: Non-graduate and graduate

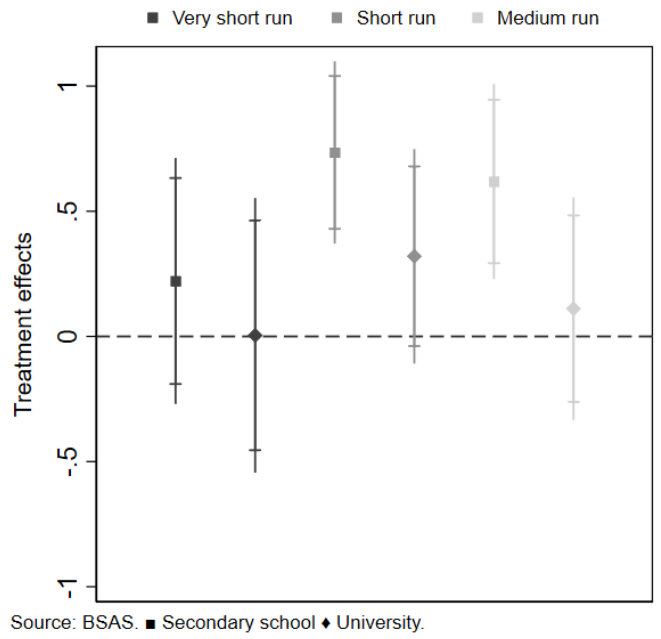




\section{Media coverage and sentiment analysis}

To empirically estimate the role of the media after the July 7th London bombings, we analysed the population of articles published in UK national newspapers on the topic of terrorism from the 31st of May to the 24th of November, preceding and following the terrorist attack. We used the Nexis Newspaper Archive which holds a deep archive of media content with coverage going back decades and including content that is no longer publicly available online. It has comprehensive news coverage for the United Kingdom with over 400 national and regional titles and is considered to have full coverage of all main newspapers from the early 2000s. We focused on the national newspapers sample which include the following list of newspapers and their online websites: the Guardian, the Telegraph, the Times (including the Sunday Times), the Financial Times, the Daily Mail (including Mail on Sunday), the Independent, the Daily Star, the Daily Express, the Daily Mirror, the Observer and the Sun. We ran a keyword search of all articles in the newspaper sample that start with any form of 'terr' (hence including 'terror', 'terrorist', 'terrorism', 'terrorists', 'terrorize'), and downloaded all the articles this keyword search identified. Sometimes the same article may appear multiple times online, all duplicates were dropped. Over the 5-month period, this was over 15000 articles. We extracted the date and time stamp, newspaper and author of each of these articles. The majority of the following text mining was done using the Tidyverse package in R.

Figure M.1 displays the mentions of "solidarity." As in the main line, the first red vertical line denotes the date of the attack, the second marks the end of the very short run, the last corresponds to the end of the short run. As can be clearly seen, "solidarity" peaks just after the attacks, potentially explaining the increase in institutional trust we observe in our data (Table 4d).

Figure M.2 looks at the mentions of other terms related to privacy and procedural rights. The picture is broadly consistent with our analysis in the main text. Words associated with security measures tend to pick in the short run relative to the very short run.

When individuals read newspaper articles, they use their understanding of the emotional intent of words to infer whether a section of text is positive or negative, or characterized by some more nuanced emotion like surprise or disgust. We can use the tools of text mining to approach the emotional content of text analytically. Our approach follows the most common approach (Silge, 2017) which is to consider the text as a combination of its individual words and the sentiment content of the whole text as the sum of the sentiment content of the individual words. The sentiment 
Figure M.1: Mentions of 'Solidarity' in UK national newspapers

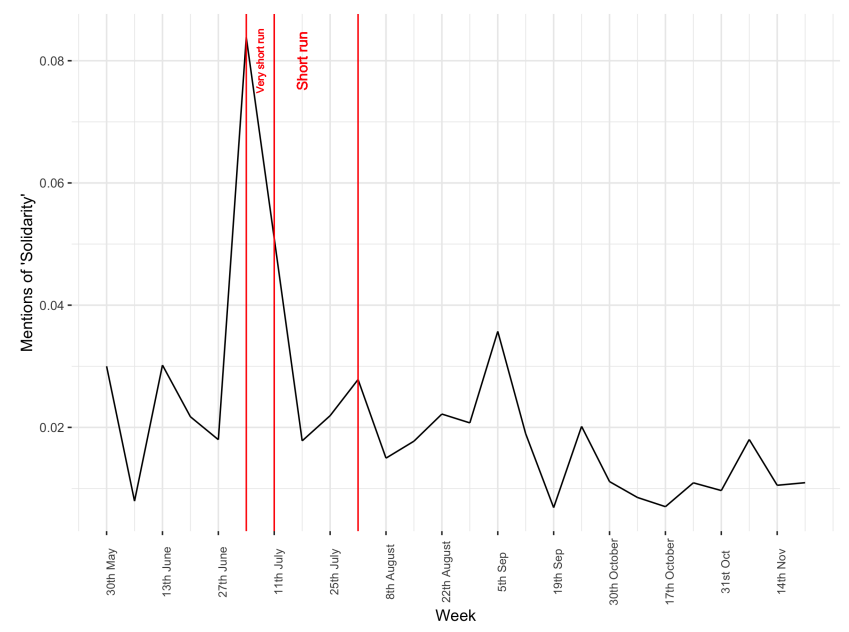

of individual words, unigrams, is defined by overlaying sentiment lexicons, one all stop words have been removed from the texts. The two general-purpose lexicons that we used in our analysis are NRC (Mohammad, 2013, Mohammad, 2015) and Bing (Bing, 2015). The Bing lexicon categorizes words in a binary fashion into positive or negative categories. The NRC lexicon categorizes words in binary categories of the following non-exclusive sentiment: positive, negative, anger, anticipation, disgust, fear, joy, sadness, surprise, and trust. These lexicons contain many English words and are trained to classify texts such as newspaper articles. We will focus on our analysis on the relative measures of sentiment, i.e. the share of words of a given sentiment in the total words of all articles published on the given day. The result of this sentiment analysis is displayed in Figure M.3. No clear pattern emerges. This tentatively suggests that the reactions we document may not be related to the sentiments expressed in the media, that is, they were not related to appeal to emotions.

Figure M.4 depicts the most frequently used words in newspaper articles on terrorism in the very short run (first week after the attack) and short run (first month minus the first week). As Figure M.4a reveals, the first week is dense of words that specifically refer to the terrorist event. "People", "attacks", "London", "train", "bus", "carriage", "bomb", "explosion", "blood", "screaming", inter alia, are the terms that recur most. The only word that could be related to privacy and procedural rights is "security". As we move forward in time, to the short run, other words become prominent. While we still find terms such as "people", "bombers", "attacks", "tube" and "train", we also observe a large number of references to "intelligence," "police," "suspect, "government," "war," "rights," "anti," etc. The word cloud depicted in Figure M.4b suggests that newspapers no longer solely focus on the attacks per se, but also consider their policy consequences and the necessary steps to prevent future attacks. 
Figure M.2: WEEKLY ARTICLES ON PRIVACY AND PROCEDURAL RIGHTS

(a) Ban

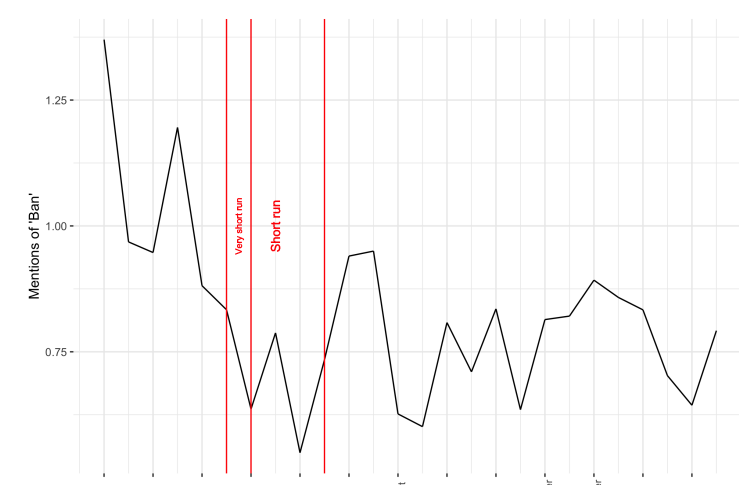

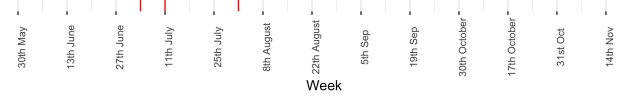

(c) Detain and Torture

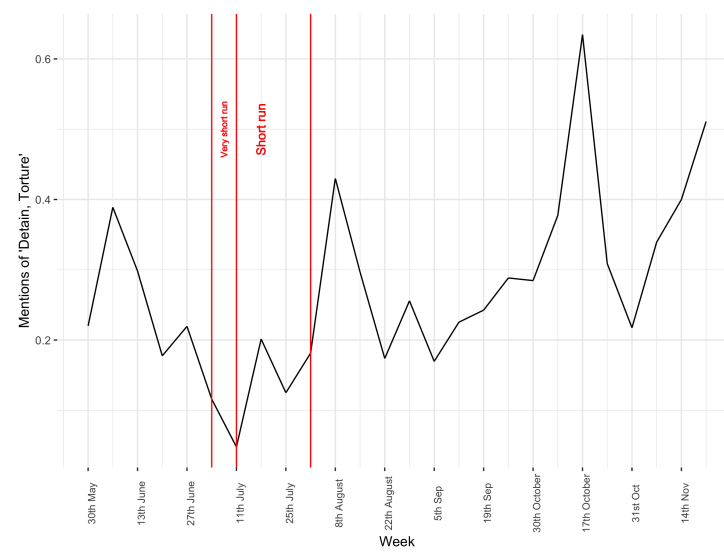

(e) Intelligence

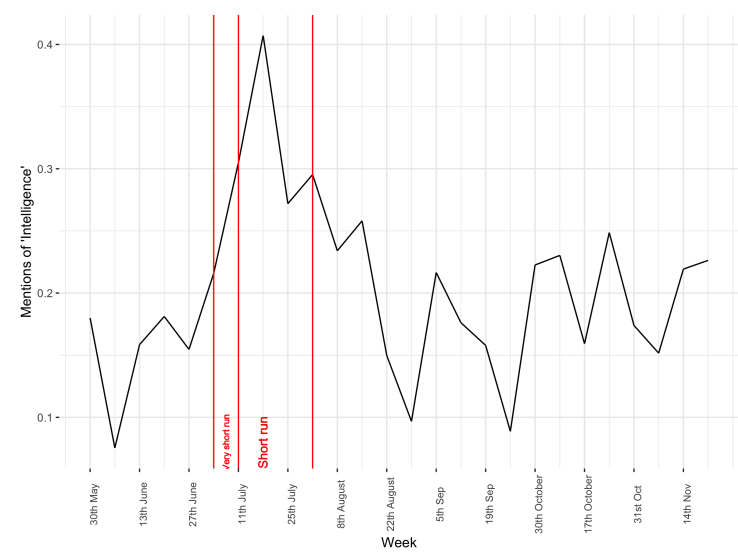

(b) ID cards

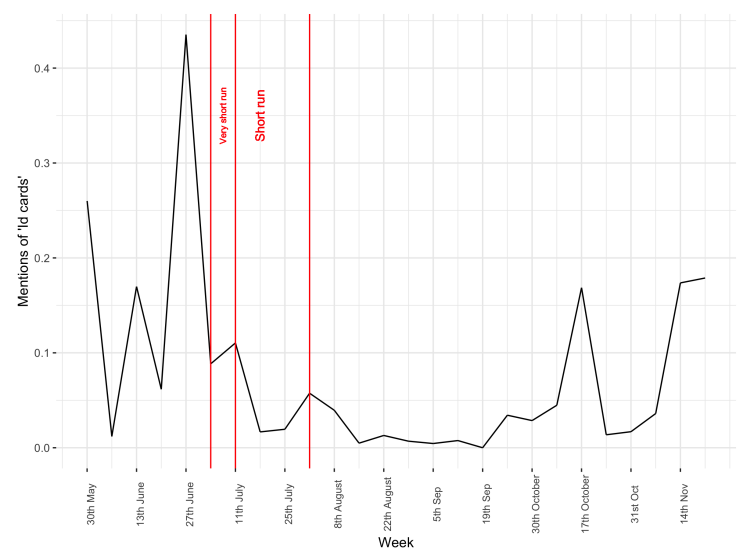

(d) Terrorism Act

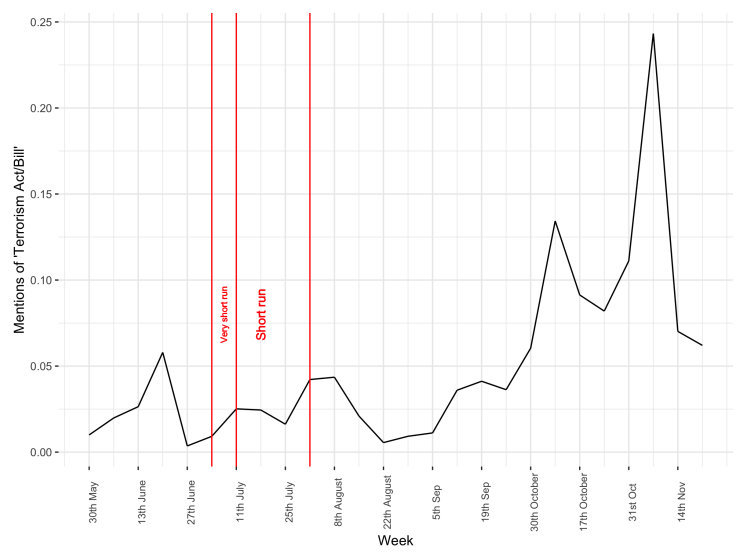

(f) Muslim

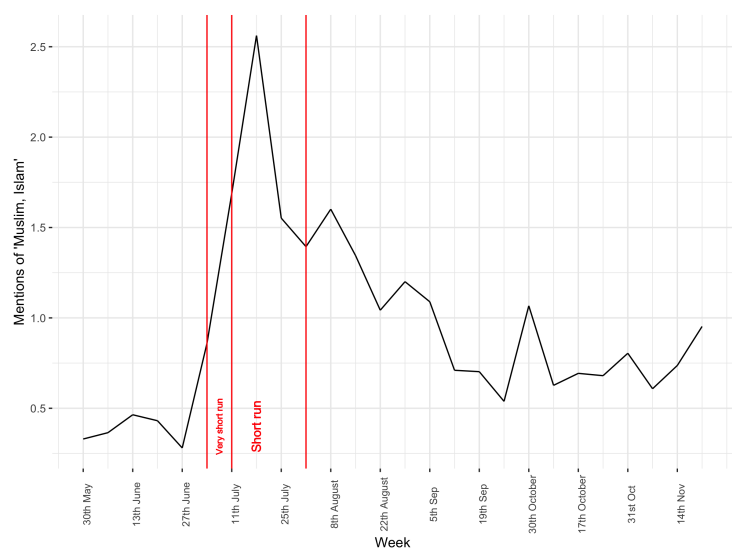


(a) Count sentiment

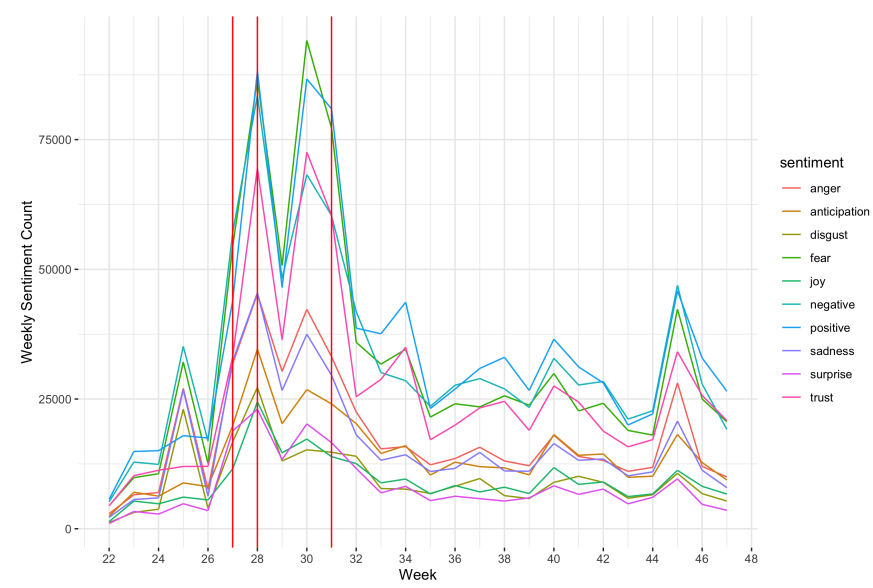

(b) Mean Sentiment

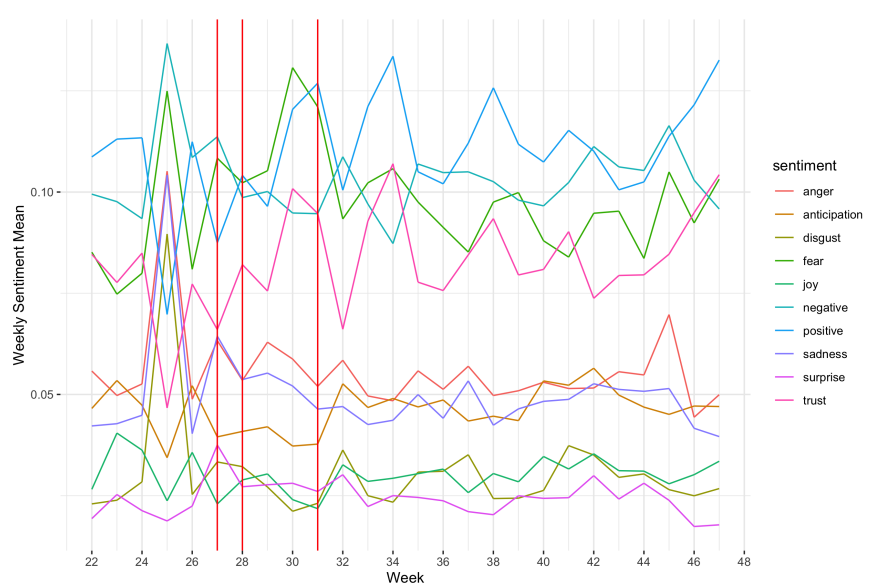

Note: This figure plots the daily measures of sentiment as calculated with the NRC lexicon. The left panel calculates the total words containing the given sentiment (anger, anticipation, disgust, fear, joy, negative, positive, sadness, surprise, trust) across all newspaper articles on terrorism that week. The right panel calculates the share of words containing the given sentiment (anger, anticipation, disgust, fear, joy, negative, positive, sadness, surprise, trust) across all words published in the all newspaper articles on terrorism that week.

Figure M.4: Word CLOUDS

(a) Very short run: first week

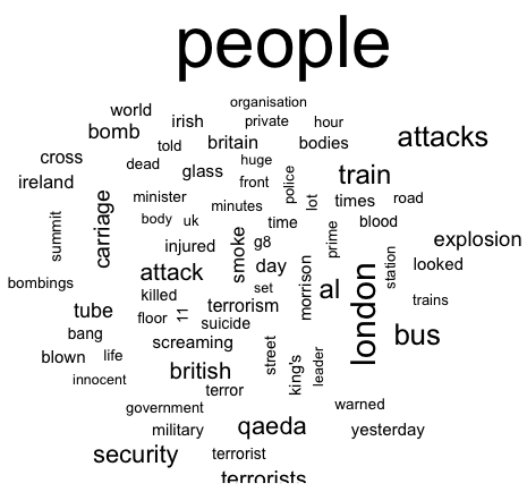

(b) Short run: first month

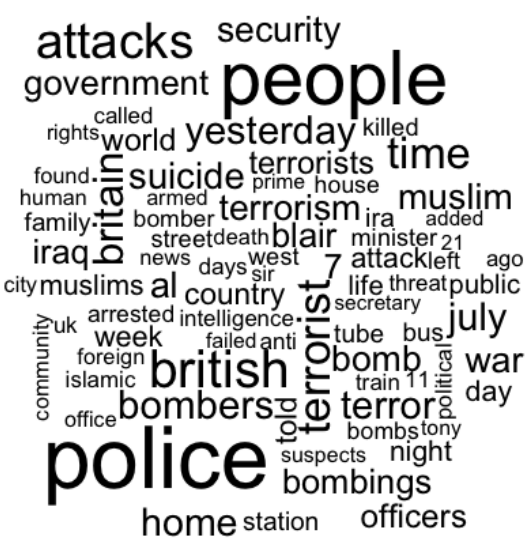




\section{References}

Bing, Liu. 2015. "Sentiment Analysis: mining sentiments, opinions, and emotions" Cambridge University Press New York.

Hainmueller, Jens. 2012. "Entropy balancing for causal effects: A multivariate reweighting method to produce balanced samples in observational studies." Political analysis, 2- (1): 25-46.

Iacus, Stefano M., Gary King, and Giuseppe Porro. 2012. "Causal inference without balance checking: Coarsened exact matching." Political analysis, 20 (1): 1-24.

Mohammad, Saif, Kiritchenko, Svetlana and Zhu, Xiaodan. 2013. "NRC-Canada: Building the state-of-the-art in sentiment analysis of tweets" arXiv preprint arXiv:1308.6242.

Mohammad, Saif M and Kiritchenko, Svetlana. 2015. "Using hashtags to capture fine emotion categories from tweets" Computational Intelligence,31(2): 301-326.

Silge, Julia, and Robinson, David. 2017. "Welcome to Text Mining with R" O'Reilly. 